\title{
ERGODIC THEOREMS WITH ARITHMETICAL WEIGHTS
}

\author{
CHRISTOPHE CUNY AND MICHEL WEBER
}

\begin{abstract}
We prove that the divisor function $d(n)$ counting the number of divisors of the integer $n$, is a good weighting function for the pointwise ergodic theorem. For any measurable dynamical system $(X, \mathcal{A}, \nu, \tau)$ and any $f \in L^{p}(\nu), p>1$, the limit

$$
\lim _{n \rightarrow \infty} \frac{1}{\sum_{k=1}^{n} d(k)} \sum_{k=1}^{n} d(k) f\left(\tau^{k} x\right)
$$

exists $\nu$-almost everywhere. We also obtain similar results for other arithmetical functions, like $\theta(n)$ function counting the number of squarefree divisors of $n$ and the generalized Euler totient function $J_{s}(n), s>0$. We use Bourgain's method, namely the circle method based on the shift model.
\end{abstract}

\section{Introduction}

Let $(X, \mathcal{A}, \nu, \tau)$ be a measurable dynamical system. Birkhoff's pointwise ergodic theorem states that for any $f \in L^{1}(\nu)$, the limit

$$
\lim _{n \rightarrow \infty} \frac{1}{n} \sum_{k=0}^{n-1} f\left(\tau^{k} x\right)=\bar{f}(x)
$$

exists $\nu$-almost everywhere and in $L^{1}(\nu)$, and $\bar{f}=\int f d \nu$ if $\tau$ is ergodic. This fundamental result was the object of many generalizations or extensions. We are interested in this article in extensions of weighted type, more particularly in extensions in which the weights are built with standard arithmetical functions, typically the divisor function $d(n)$, counting the number of divisors of the integer $n$. This is the most standard example of multiplicative arithmetical function, but the reason to focus on this particular type of weights lies on deeper considerations.

First introduce the necessary notation. For a sequence $\left(w_{k}\right)_{k \geq 1}$ of real numbers (weights) such that $W_{n}:=\sum_{k=1}^{n}\left|w_{k}\right| \neq 0$ and $W_{n} \rightarrow \infty$, we define the weighted averages

$$
A_{n}^{\tau} f:=\frac{1}{W_{n}} \sum_{k=1}^{n} w_{k} f \circ \tau^{k} .
$$

We are interested in their almost everywhere convergence.

As we will see later on (Theorems 2.6, 2.9, 3.7, Proposition 2.14 and Remark 2.7), elementary considerations based on Hopf's maximal Lemma or on properties of Dirichlet convolution products allow to directly derive from Birkhoff's theorem, weighted extensions where the weights can be either of the examples below

$$
\begin{aligned}
\omega(k), \Omega(k) & \text { the prime divisor function and the sum of prime divisor function, } \\
\sigma_{s}(k) & \text { the sum of } s \text {-powers of divisors of } k, s \neq 0 .
\end{aligned}
$$

The case of the divisor function corresponding to the second example with $s=0$, does not seem to be reduced to this approach. Another motivation lies in a recent result of Berkes, Müller and Weber [2], Theorem 3.

Theorem A. Let $f$ be a non-negative multiplicative function and let $F(n)=\sum_{k=1}^{n} f(k), n \geq 1$. Assume that there are positive constants $C_{1}, C_{2}, C_{3}, C_{4}$ and $a>1$ such that
(i) $\sum_{p \leq x} f(p)^{a} \log p \leq C_{1} x$,
(ii) $\sum_{p, \nu \geq 2} \frac{f\left(p^{\nu}\right)^{a} \log p^{\nu}}{p^{\nu}} \leq C_{2}$,
(iii) $\sum_{p \leq x} f(p) \log p \geq C_{3} x$ for $x \geq C_{4}$. 
Let $X_{1}, X_{2}, \ldots$ be i.i.d. integrable random variables. Then $\lim _{n \rightarrow \infty} \frac{1}{F(n)} \sum_{k=1}^{n} f(k) X_{k} \stackrel{a . s .}{=} \mathbb{E} X_{1}$.

As a consequence, the weighted strong law of large numbers holds with $f(n)=d(n)$ (and even for $f(n)=d\left(n^{l}\right)^{\alpha}$ with $l \geq 1$ integer and real $\left.\alpha>0\right)$. Theorem $\mathrm{A}$ is obtained by showing that Jamison, Orey and Pruitt combinatorial criterion is satisfied under the above set of conditions.

Here, we use Bourgain's method to study the case of the divisor function. As Bourgain mentionned in 4], his method (the circle method on the shift model) is general and should apply as soon as one has a good control on the exponential sums inherent to the problem. One can then replace the Fourier kernels appearing in the problem considered by more regular ones with a suitable control on the error term.

We say that $\left(w_{k}\right)_{k \geq 1}$ is a good weight for the dominated ergodic theorem in $L^{p}, p>1$, if there exists $C_{p}>0$ such that for every (ergodic) dynamical system $(X, \mathcal{A}, \nu, \tau)$ and every $f$ in $L^{p}$,

$$
\left\|\sup _{n \geq 1} \frac{\left|\sum_{1 \leq k \leq n} w_{k} f \circ \tau^{k}\right|}{W_{n}}\right\|_{p} \leq C_{p}\|f\|_{p} .
$$

We also say that $\left(w_{k}\right)_{k \geq 1}$ is a good weight for the pointwise ergodic theorem in $L_{p}, p>1$, if for every (ergodic) dynamical system $(X, \mathcal{A}, \nu, \tau)$ and every $f$ in $L^{p},\left(\left(\sum_{1<k<n} w_{k} f \circ \tau^{k}\right) / W_{n}\right)_{n}$ converges $\nu$-a.s. Alternatively, when the weights are generated by an arithmetical function $w$, we say that $w$ is a good weighting function.

We now state our main result.

Theorem 1.1. The divisor function is a good weighting function for both the dominated and the pointwise ergodic theorem in $L_{p}, p>1$.

Using properties of the Dirichlet convolution, it will also follow that the above result remains true for other arithmetical weights. Let $\theta(n)$ be the multiplicative function counting the number of squarefree divisors of $n$, and let $J_{s}(n)$ be the generalized Euler totient function. Recall that $\theta(k)=2^{\omega(k)}$ and $J_{s}(n)=\sum_{d \mid n} d^{s} \mu\left(\frac{n}{d}\right)$ where $\mu$ is Möbius function.

Theorem 1.2. The $\theta$ function and generalized Euler totient function $J_{s}, s>0$, the sum of $s$-powers of divisor function $\sigma_{s}, s \neq 0$ and the modulus of Möbius function are good weighting functions for both the dominated and the pointwise ergodic theorem in $L_{p}, p>1$.

The paper is organized as follows. In Section 2. we derive from the dominated and the pointwise ergodic theorem (in $L^{p}, p>1$ ) several weighted ergodic theorems where the weights are mainly additive arithmetical functions. We use a Theorem of Delange [8] and the Turán-Kubilius inequality (see e.g. [16]), as well as the result of Davenport and of Bateman and Chowla for the case of the Möbius and Liouville functions respectively.

In Section 3, we consider the following problem. Given a good weighting function $a$ and another arithmetical function $b$, we study the conditions under which the Dirichlet convoluted function $a * b$ is again a good weighting function. We recall that $a * b$ is defined by $a * b(n)=\sum_{d \mid n} a(d) b(n / d)$. After having first proved some lemmas a bit in the spirit of Wintner's theorem, we obtain in Proposition 3.3, a general condition showing a kind of conservation property for the dominated ergodic theorem under the action of the Dirichlet convolution product. We next apply it and show that the sum of $s$-powers of divisors of $k, s \neq 0$, the number of squarefree divisors of $k$, the generalized Euler totient function and the modulus of Möbius function are good weighting functions for the dominated ergodic theorem in $L^{p}, p>1$, and for the pointwise ergodic theorem in $L^{p}, p \geq 1$.

In section 4, Bourgain's approach is briefly described, essentially the key steps are presented. In section 5, several estimates concerning the divisor exponential sums $D_{n}(x)=\sum_{1<k<n} d(k) \mathrm{e}^{2 i k \pi x}$ are proved, depending on the proximity of $x$ to rationals with small or large denominators. We proceed rather simply and will not for instance use Voronoï identity, nor need elaborated estimates.

In the two next sections, we apply Bourgain's approach. In Section 6, we use Fourier analysis to establish maximal inequalities related to auxiliary kernels. In Section 7 we explain how to derive 
good approximation results with suitable Fourier kernels to which we can apply the previous maximal inequalities. Theorem 1.1 is proved in section 8 ,

\section{Derivation Results from Birkhoff's Theorem.}

For a sequence $\left(a_{n}\right)_{n \geq 1}$, write $A_{n}^{(m)}=\sum_{k=1}^{n} a_{k}^{m}$, for every real number $m>0$. Define also $\tilde{A}_{n}:=A_{n} / n$. The following is a basic application of Hölder inequality, hence the proof is omitted.

Lemma 2.1. Let $\left(a_{n}\right)_{n \geq 1}$ be a sequence of non-negative numbers. Assume that there exists $C>0$ and $m>1$ such that for every $n \geq 1, \sum_{k=1}^{n} a_{k}^{m} \leq C n \tilde{A}_{n}^{m}$. Then, for every $1 \leq s \leq m$ and every $n \geq 1, \sum_{k=1}^{n} a_{k}^{s} \leq C_{m, s} n \tilde{A}_{n}^{s}$, with $C_{m, s}=C^{m(s-1) /(s(m-1))}$.

We deduce the following automatic dominated ergodic theorem.

Lemma 2.2. Let $\left(a_{n}\right)_{n \geq 1}$ be a sequence of non-negative numbers. Assume that there exists $C>0$ and $m>1$ such that for every $n \geq 1, \sum_{k=1}^{n} a_{k}^{m} \leq C n \tilde{A}_{n}^{m}$. Then, for every $r>m /(m-1)$, $\left(a_{n}\right)_{n \geq 1}$ is good for the dominated ergodic theorem in $L^{r}$.

Remark 2.3. It follows from the proof that, for $r=m /(m-1)$, there is a dominated ergodic theorem of weak-type.

Proof. Let $(X, \mathcal{A}, \nu, \tau)$ be a dynamical system and let $f \in L^{r}(\nu)$, for some $r \in(m /(m-1),+\infty]$. Then, $r /(r-1)<m$. Now let $r /(r-1)<s<m$. Then, writing $s^{\prime}:=s /(s-1)$, we have $m /(m-1)<s^{\prime}<r$.

By Lemma 2.1, we have $\sum_{k=1}^{n} a_{k}^{s} \leq C n \tilde{A}_{n}^{s}$. By Hölder inequality, we have

$$
\begin{aligned}
\left|\sum_{k=1}^{n} a_{k} f \circ \tau^{k}\right| & \leq\left(\sum_{k=1}^{n} a_{k}^{s}\right)^{1 / s}\left(\sum_{k=1}^{n}\left|f \circ \tau^{k}\right|^{s^{\prime}}\right)^{1 / s^{\prime}} \leq C^{1 / s} n^{1 / s} \tilde{A}_{n}\left(\sum_{k=1}^{n}\left|f \circ \tau^{k}\right|^{s^{\prime}}\right)^{1 / s^{\prime}} \\
& =C^{1 / s} A_{n}\left(\frac{1}{n} \sum_{k=1}^{n}\left|f \circ \tau^{k}\right|^{s^{\prime}}\right)^{1 / s^{\prime}} .
\end{aligned}
$$

Corollary 2.4. Let $\left(a_{n}\right)_{n \geq 1}$ be a sequence of non-negative numbers. Assume that there exists $m>1$ such that

$$
\sum_{k=1}^{n}\left|a_{k}-\tilde{A}_{n}\right|^{m}=o\left(n \tilde{A}_{n}^{m}\right) .
$$

Then, for every $r>m /(m-1),\left(a_{n}\right)_{n \geq 1}$ is good for both the dominated and the pointwise ergodic theorem in $L^{r}$.

Remark 2.5. It follows from the proof that, for $s=m /(m-1)$, there is a dominated ergodic theorem of weak-type and a pointwise weighted ergodic theorem.

Proof. Using that $a_{k}^{m} \leq 2^{m-1}\left(\tilde{A}_{n}^{m}+\left|a_{k}-\tilde{A}_{n}\right|^{m}\right)$, we see that there exists $C>0$ such that for every $n \geq 1 \sum_{k=1}^{n} a_{k}^{m} \leq C n \tilde{A}_{n}^{m}$, and Lemma 2.2 applies, as well as the remark after it. By the Banach principle, we just have to prove the pointwise convergence for bounded functions. Let $(X, \mathcal{A}, \nu, \tau)$ be a dynamical system and let $f \in L^{\infty}(\nu)$. Let $K \geq 0$ be such that $f \leq K \nu$-a.s.

We have, by Hölder

$$
\left|\sum_{k=1}^{n}\left(a_{k}-\tilde{A}_{n}\right) f \circ \tau^{k}\right| \leq K n^{1-1 / m}\left(\sum_{k=1}^{n}\left|a_{k}-\tilde{A}_{n}\right|^{m}\right)^{1 / m}=o\left(n \tilde{A}_{n}\right),
$$

and the result follows.

Theorem 2.6. Let $(g(n))_{n \geq 1}$ be an additive function with values in $\mathbb{N}$ and such that $g(p)=1$ for every prime number $p$. Assume moreover that there exists $\beta>0$, such that for every $\nu \geq 1$ and every prime number $p$,

$$
g\left(p^{\nu}\right) \leq \beta \nu \log p
$$


Then $(g(n))_{n \geq 1}$ is a good weight for both the dominated and pointwise ergodic theorem in $L^{p}$, $p>1$.

Remark 2.7. It follows from the proof that for every real number $m \geq 1,\left(g(n)^{m}\right)_{n \geq 1}$ is a good weight for the dominated ergodic theorem in $L^{p}, p>1$. When $m$ is an integer, it is also a good weight for the pointwise ergodic theorem in $L^{p}, p>1$. The theorem applies in particular with $g(n)=\omega(n)$ and $g(n)=\Omega(n)$

Proof. Let us recall the following corollary of a deep result of Delange [8]. The corollary corresponds to Theorem 2 (p. 132) with $\nu=m$ and $\chi \equiv 1$, provided that (9) in [8] be satisfied. We shall check this below.

Theorem 2.8. Let $(g(n))_{n \geq 1}$ be as in Corollary [2.6. For every integer $m \geq 1$, we have

$$
\left.\sum_{1 \leq n \leq x} g(n)^{m}=x(\log \log x)^{m}+O\left(x(\log \log x)^{m-1}\right)\right) .
$$

We see that the assumptions of Lemma 2.1 (hence of Lemma 2.2) are satisfied for every integer $m \geq 1$, with $\alpha=0$. Hence we have the dominated ergodic theorem.

Let us prove the pointwise convergence of the weighted averages. It suffices to prove the convergence for bounded functions. Let $(X, \mathcal{A}, \nu, \tau)$ be a dynamical system. Let $f \in L^{\infty}(\nu)$, with $|f| \leq A$. We agree to denote here and in what follows $\log \log x=\log (\log (2+x)), x \geq 1$. We have

$$
\sum_{n=1}^{N} g(n) f \circ \tau^{n}=(\log \log N) \sum_{n=1}^{N} f \circ \tau^{n}+\sum_{n=1}^{N}(g(n)-\log \log N) f \circ \tau^{n} .
$$

By Theorem 2.8 and Birkhoff's ergodic theorem, $\frac{\log \log N}{\sum_{1 \leq k \leq N} g(k)} \sum_{1 \leq k \leq N} f \circ \tau^{k}$ converges $\nu$-a.s. To conclude it suffices to prove that the second term in (2.2) converges $\nu$-a.s to 0 .

By Cauchy-Schwarz's inequality, we have

$$
\left|\sum_{n=1}^{N}(g(n)-\log \log N) f \circ \tau^{n}\right| \leq A \sqrt{N}\left(\sum_{n=1}^{N}(g(n)-\log \log N)^{2}\right)^{1 / 2}
$$

Using Theorem 2.8 with $m=1$ and $m=2$ and $(g(n)-\log \log N)^{2}=g(n)^{2}-2 g(n) \log \log N+$ $(\log \log N)^{2}$, we see that there exists $C>0$ such that

$$
\left|\sum_{n=1}^{N}(g(n)-\log \log N) f \circ \tau^{n}\right| \leq C \sqrt{N}(N \log \log N)^{1 / 2}=o\left(\sum_{1 \leq n \leq N} g(k)\right),
$$

and the proof is completed.

Let us prove under (2.1) that the condition (9) of [8] is satisfied. We have to prove that there exists $\rho>1$ and $\sigma<1$ such that $\sum_{k \geq 2, p \in \mathcal{P}} \frac{\rho^{g\left(p^{k}\right)}}{p^{\sigma k}}<\infty$. Take $\sigma=3 / 4$ and $\rho>1$ such that $\gamma:=2(\sigma-\beta \log \rho)>1$. Notice that

$$
\sum_{k \geq 2} \frac{\rho^{g\left(p^{k}\right)}}{p^{\sigma k}} \leq \sum_{k \geq 2}\left(\frac{\rho^{\beta \log p}}{p^{\sigma}}\right)^{k} \leq \frac{1}{p^{\gamma}} \frac{1}{1-1 / p^{\gamma / 2}} \leq \frac{1}{p^{\gamma}} \frac{1}{1-1 / 2^{\gamma / 2}},
$$

and the desired result follows.

Theorem 2.9. Let $g(n)_{n \geq 1}$ be an additive function such that

$$
\left(\sum_{p^{\alpha} \leq n} \frac{g\left(p^{\alpha}\right)^{2}}{p^{\alpha}}\right)^{1 / 2}=o\left(\sum_{p^{\alpha} \leq n} \frac{g\left(p^{\alpha}\right)}{p^{\alpha}}\right) .
$$

Then, $\left(g(n)_{n \geq 1}\right.$ is good for both the dominated and the pointwise ergodic theorem in $L^{p}$ for every $p>2$. 
Proof. Recall the Turán-Kubilius inequality [16] p. 302. There exists an absolute constant $\widetilde{C}$ such that for any additive complex-valued arithmetic function $f$,

$$
\frac{1}{n} \sum_{k=1}^{n}\left|f(k)-\sum_{p^{\alpha} \leq n} \frac{f\left(p^{\alpha}\right)}{p^{\alpha}\left(1-p^{-1}\right)}\right|^{2} \leq \widetilde{C} \sum_{p^{\alpha} \leq n} \frac{\left|f\left(p^{\alpha}\right)\right|^{2}}{p^{\alpha}}, \quad(n \geq 2) .
$$

Let $\gamma(n)=\sum_{p^{\alpha} \leq n} \frac{g\left(p^{\alpha}\right)}{p^{\alpha}\left(1-p^{-1}\right)}$. By Cauchy-Schwarz's inequality, next Turán-Kubilius inequality,

$$
\left|\frac{1}{n} \sum_{k=1}^{n}(g(k)-\gamma(n))\right|^{2} \leq \frac{1}{n} \sum_{k=1}^{n}|g(k)-\gamma(n)|^{2} \leq C \sum_{p^{\alpha} \leq n} \frac{\left|g\left(p^{\alpha}\right)\right|^{2}}{p^{\alpha}}=o\left(|\gamma(n)|^{2}\right) .
$$

In particular, $\widetilde{G}(n)=\frac{1}{n} \sum_{k=1}^{n} g(k)=\gamma(n)+H$, where $H=o(|\gamma(n)|)$. Writing $H=h \gamma(n)$ with $|h| \leq 1 / 2$, if $n$ is large, we have $|\gamma(n)| \leq|\widetilde{G}(n)| /(1-|h|) \leq 2|\widetilde{G}(n)|$, and by Minkowski's inequality,

$$
\left(\frac{1}{n} \sum_{k=1}^{n}|g(k)-\widetilde{G}(n)|^{2}\right)^{1 / 2}=o(|\gamma(n)|)=o(|\widetilde{G}(n)|) .
$$

We conclude by applying Corollary 2.4.

The case of Möbius and Liouville functions. Here we consider the $\nu$-a.s. behaviour of the sums $\sum_{k=1}^{n} \mu(k) f \circ \tau^{k}, \sum_{k=1}^{n} \lambda(k) f \circ \tau^{k}$ where $\mu$ is the Möbius function and $\lambda$ is Liouville function. We only treat the case of the Möbius function, the arguments being quite identical for the Liouville function.

Let us recall the following result of Davenport [6] on the behaviour of the corresponding exponential sums.

Proposition 2.10. For every $h>0$ there exists $C_{h}>0$ such that

$$
\sup _{x \in[-1 / 2,1 / 2]}\left|\sum_{k=1}^{n} \mu(k) \mathrm{e}^{2 i \pi k x}\right| \leq \frac{C_{h} n}{(\log n)^{h}} .
$$

Remark 2.11. According to Lemma 1 in Bateman and Chowla [1, an analog estimate holds for the Liouville function.

By the spectral theorem (see e.g. [17, Proposition 1.2.2), we easily deduce the following.

Corollary 2.12. For every $h>0$, there exists $C_{h}>0$ such that for every $f \in L^{2}(\nu)$

$$
\left\|\sum_{k=1}^{n} \mu(k) f \circ \tau^{k}\right\|_{2} \leq \frac{C_{h} n}{(\log n)^{h}}\|f\|_{2} .
$$

Notice that, trivially, for $f \in L^{p}(\nu), 1 \leq p \leq \infty$, we have $\left\|\sum_{k=1}^{n} \mu(k) f \circ \tau^{k}\right\|_{p} \leq n\|f\|_{p}$. Hence, performing interpolation between $L^{1}(\nu)$ and $L^{2}(\nu)$ on the one hand and between $L^{2}(\nu)$ and $L^{\infty}(\nu)$, on the other hand, we easily derive the following.

Corollary 2.13. For every $h>0$ and every $p>1$, there exists $C_{h, p}>0$ such that or every $f \in L^{p}(\nu)$

$$
\left\|\sum_{k=1}^{n} \mu(k) f \circ \tau^{k}\right\|_{p} \leq \frac{C_{h, p} n}{(\log n)^{h}}\|f\|_{p}
$$

It is mentionned by Sarnak [14 that Bourgain's approach allows to prove that for every $f \in$ $L^{2}(\nu), \frac{1}{n} \sum_{k=1}^{n} \mu(k) f \circ \tau^{k} \underset{n \rightarrow \infty}{\longrightarrow} 0, \nu$-a.s. In view of (2.3), one could wonder whether we have a rate in this $\nu$-a.s. convergence. We shall prove the following.

Proposition 2.14. For every $h>0$ and every $p>1$ there exists $C_{h, p}>0$ such that for every $f \in L^{p}(\nu)$,

$$
\left\|\sup _{n \geq 1} \frac{\left|\sum_{k=1}^{n} \mu(k) f \circ \tau^{k}\right|}{n /(\log n)^{h}}\right\|_{p} \leq C_{h, p}\|f\|_{p} .
$$

In particular, for every $h>0, \frac{(\log n)^{h}}{n} \sum_{k=1}^{n} \mu(k) f \circ \tau^{k} \underset{n \rightarrow \infty}{\longrightarrow} 0$. 
Proof. Let $p>1$ and $h>0$. Let $0<\varepsilon<\frac{p-1}{p(1+h)}$. Let $h^{\prime}>h+1 / \varepsilon$. Let $f \in L^{p}(\nu)$. Denote

$$
M_{n}=M_{n, h}(f):=\frac{(\log n)^{h}}{n} \sum_{k=1}^{n} \mu(k) f \circ \tau^{k} .
$$

Denote also $u_{n}:=\left[\mathrm{e}^{n^{\varepsilon}}\right]$. By Corollary 2.13, there exists $C_{h^{\prime}, p}$ such that, for every $n \geq 1$,

$$
\left\|M_{n}\right\|_{p} \leq \frac{C_{h^{\prime}, p}}{(\log n)^{h^{\prime}-h}}\|f\|_{p}
$$

In particular, we see that

$$
\left\|\sup _{n \geq 1}\left|M_{u_{n}}\right|\right\|_{p}^{p} \leq \sum_{n \geq 1}\left\|M_{u_{n}}\right\|_{p}^{p} \leq C\|f\|_{p}^{p} \sum_{n \geq 1} \frac{1}{n^{p \varepsilon\left(h^{\prime}-h\right)}},
$$

and the latter series converges by our choice of $h^{\prime}$. Now let $n \geq 1$ and $u_{n}<m \leq u_{n+1}$. Write $m=u_{n}+k$. We have, writing $\sum_{i=1}^{m}=\sum_{i=1}^{u_{n}}+\sum_{i=u_{n+1}}^{m}$

$$
\left|M_{m}\right| \leq\left|M_{u_{n}}\right|+\frac{C n^{\varepsilon h}}{u_{n}} \sum_{i=u_{n}+1}^{u_{n+1}}|f| \circ \tau^{i}
$$

Hence,

$$
\begin{aligned}
\max _{u_{n}<m \leq u_{m+1}}\left|M_{m}\right| & \leq\left|M_{u_{n}}\right|+\frac{C n^{\varepsilon h}}{u_{n}} \sum_{i=u_{n}+1}^{u_{n+1}}|f| \circ \tau^{i} \\
& \leq \sup _{\ell \geq 1}\left|M_{u_{\ell}}\right|+C\left(\sum_{\ell \geq 1}\left(\frac{\ell^{\varepsilon h}}{u_{\ell}} \sum_{i=u_{\ell}+1}^{u_{\ell+1}}|f| \circ \tau^{i}\right)^{p}\right)^{1 / p} .
\end{aligned}
$$

Now, using that $u_{\ell+1}-u_{\ell}=\mathcal{O}\left(u_{\ell} / \ell^{1-\varepsilon}\right)$, we see that there exists $C>0$ such that

$$
\left\|\sum_{i=u_{\ell}+1}^{u_{\ell+1}}|f| \circ \tau^{i}\right\|_{p} \leq \frac{C u_{\ell}\|f\|_{p}}{\ell^{1-\varepsilon}}
$$

Hence

$$
\left\|\sup _{m \geq 1}\left|M_{m}\right|\right\|_{p} \leq\left\|\sup _{\ell \geq 1}\left|M_{u_{\ell}}\right|\right\|_{p}+C\|f\|_{p}\left(\sum_{\ell \geq 1} \frac{1}{\ell p(1-\varepsilon(1+h))}\right)^{1 / p}
$$

and the desired result follows since $p(1-\varepsilon(1+h))>1$.

\section{Ergodic stability of the Dirichlet convolution.}

Let us recall the following basic fact. Let $a(n)$ and $b(n)$ be two arithmetical functions with summatory functions $A(x)=\sum_{n \leq x} a(n)$ and $B(x)=\sum_{n \leq x} b(n)$. Then

$$
\sum_{n \leq x} a * b(n)=\sum_{n \leq x} a(n) B\left(\frac{x}{n}\right)=\sum_{n \leq x} b(n) A\left(\frac{x}{n}\right) .
$$

Recall that a function $f:[0,+\infty) \rightarrow(0,+\infty)$ is slowly varying if for every $K>0$,

$$
\lim _{x \rightarrow+\infty} \frac{f(K x)}{f(x)}=0 .
$$

We start with a lemma a bit in the spirit of Wintner's theorem [20] p. 180, and that should be known from specialists in number theory.

Lemma 3.1. Let $a(n)$ be a non-negative arithmetic function such that $A(x) \sim x^{\alpha} L(x)$ as $x \rightarrow \infty$, for some $\alpha>0$ and some positive non-decreasing slowly varying function $L$. Let $b(n)$ be an arithmetic function such that $\sum_{n \geq 1} \frac{|b(n)|}{n^{\alpha}}<\infty$. Then

$$
\lim _{n \rightarrow \infty} \frac{1}{A(n)} \sum_{k=1}^{n} b * a(k)=\sum_{m=1}^{\infty} \frac{b(m)}{m^{\alpha}} .
$$


Proof. Denote $c(k)=b * a(k)$. Let $M \geq 1$ be an integer fixed for the moment. By assumption $x:=\sum_{m \geq 1} \frac{b(m)}{m^{\alpha}}$ is well defined. Denote also $x_{M}:=\sum_{m \geq M+1} \frac{b(m)}{m^{\alpha}}$.

$$
\left|x-\sum_{1 \leq k \leq n} c(k) / A(n)\right| \leq \sum_{1 \leq \ell \leq M}\left|\frac{b(\ell)}{\ell^{\alpha}}-\frac{b(\ell) A(n / \ell)}{A(n)}\right|+\left|x_{M}\right|+\sum_{M<\ell \leq n}|b(\ell)| A(n / \ell) / A(n) .
$$

By assumption, for every $1 \leq \ell \leq M, A(n / \ell) / A(n) \underset{n \rightarrow \infty}{\longrightarrow} 1 / \ell^{\alpha}$. Since $L$ is positive and nondecreasing, there exists $C>0$ such that,

$$
\begin{aligned}
A(x) & \leq C x^{\alpha} L(x) & \forall x \geq 1, \\
A(n / \ell) & \leq C \frac{n^{\alpha} L(n)}{\ell^{\alpha}} & \forall n \geq 1, \forall 1 \leq \ell \leq n .
\end{aligned}
$$

Hence $\sum_{M<\ell \leq n}|b(\ell)| A(n / \ell) / A(n) \leq C \frac{n^{\alpha} L(n)}{A(n)} \sum_{M<\ell \leq n}|b(\ell)| / \ell^{\alpha}$, and so

$$
\limsup _{n \rightarrow \infty}\left|\sum_{1 \leq k \leq n} \frac{c(k)}{A(n)}-x\right| \leq\left|x_{M}\right|+C \sum_{\ell>M} \frac{|b(\ell)|}{\ell^{\alpha}} .
$$

As the right-term tends to 0 when $M$ tends to infinity, this proves the result.

Lemma 3.2. Let $a(n)$ be a non-negative arithmetic function such that $A(n) \sim n^{\alpha} /(\log n)^{\beta}$ for some $\alpha, \beta>0$. Let $b(n)$ be an arithmetic function such that $\sum_{n \geq 1} \frac{|b(n)|(\log n)^{\beta}}{n^{\alpha}}<\infty$. Then

$$
\lim _{n \rightarrow \infty} \frac{1}{A(n)} \sum_{k=1}^{n} b * a(k)=\sum_{m=1}^{\infty} \frac{b(m)}{m^{\alpha}} .
$$

Proof. We proceed as above, using the same notation. Let $M \geq 1$ be a an integer fixed for the moment. We have

$$
\begin{aligned}
\left|x-\sum_{1 \leq k \leq n} \frac{c(k)}{A(n)}\right| \leq & \sum_{1 \leq \ell \leq M}\left|\frac{b(\ell)}{\ell^{\alpha}}-\frac{b(\ell) A(n / \ell)}{A(n)}\right|+\left|x_{M}\right| \\
& +\sum_{M<\ell \leq \sqrt{n}}|b(\ell)| A(n / \ell) / A(n)+\sum_{\sqrt{n}<\ell \leq n}|b(\ell)| A(n / \ell) / A(n) .
\end{aligned}
$$

Now,

$$
\sum_{M<\ell \leq \sqrt{n}}|b(\ell)| A(n / \ell) / A(n) \leq C \sum_{\ell>M}|b(\ell)| / \ell^{\alpha},
$$

and

$$
\sum_{\sqrt{n}<\ell \leq n}|b(\ell)| A(n / \ell) / A(n) \leq(\log n)^{\beta} \sum_{\ell>\sqrt{n}}|b(\ell)| / \ell^{\alpha} \underset{n \rightarrow \infty}{\longrightarrow} 0,
$$

by a result analogue to the Kronecker lemma. Then we conclude as above.

Proposition 3.3. Let $a(n)$ be a non-negative arithmetic function such that $A(n) \sim n^{\alpha} L(n)$ for some $\alpha>0$ and some non-decreasing slowly varying function $L$. Let $b(n)$ be an arithmetic function such that $\sum_{n \geq 1}|b(n)| / n^{\alpha}<\infty, \sum_{n \geq 1} b(n) / n^{\alpha} \neq 0$ and $a * b(n) \geq 0$ for every $n \geq 1$. Let $p>1$.

(i) Assume that a(n) satisfies to the dominated ergodic theorem in $L^{p}$. Then, $a * b(n)$ satisfies to the dominated ergodic theorem either.

(ii) If moreover, a(n) satisfies to the pointwise ergodic theorem in $L^{p}$ then $a * b(n)$ satisfies to the pointwise ergodic theorem either.

Remark 3.4. If $A(n) \sim n^{\alpha} /(\log n)^{\beta}$, for some $\beta>0$, then the conclusion of the theorem holds as soon as $\sum_{n \geq 1}|b(n)|(\log n)^{\beta} / n^{\alpha}<\infty$ and $\sum_{n \geq 1} b(n) / n^{\alpha} \neq 0$. When the pointwise ergodic theorem holds, the limit may be identified for the weigth $a * b(n)$ whenever it is identified for the weight $a(n)$. 
Proof. Let $(X, \mathcal{A}, \nu, \tau)$ be a dynamical system. Let $f \in L^{p}(\nu)$. By Lemma 3.1, it suffices to prove a maximal inequality and the almost-everyhere convergence for $\left(\frac{\sum_{1 \leq k \leq n} c(k) f \circ \tau^{k}}{A(n)}\right)_{n \geq 1}$, where, as before, $c(n)=a * b(n)$.

Let us prove $(i)$. Write

$$
\mathcal{A}_{\ell}=\mathcal{A}_{\ell}(f)=\sup _{n \geq 1} \frac{\left|\sum_{1 \leq k \leq n} a(k) f \circ \tau^{\ell k}\right|}{A(n)} .
$$

By assumption, there exists $C>0$ (independent on $\ell$ anf $f$ ) such that

$$
\left\|\mathcal{A}_{\ell}\right\|_{p, \nu} \leq C\|f\|_{p, \nu} .
$$

Using (3.1), we see that

$$
\begin{aligned}
\frac{\left|\sum_{1 \leq k \leq n} c(k) f \circ \tau^{k}\right|}{A(n)} & \leq \frac{\sum_{1 \leq \ell \leq n} b(\ell)\left|\sum_{1 \leq k \leq n / \ell} a(k) f \circ \tau^{\ell k}\right|}{A(n)} \\
& \leq \frac{\sum_{1 \leq \ell \leq n} b(\ell) A(n / \ell) \mathcal{A}_{\ell}}{A(n)} \leq C \sum_{\ell \geq 1} \frac{|b(\ell)|}{\ell^{\alpha}} \mathcal{A}_{\ell} .
\end{aligned}
$$

and we deduce the desired maximal inequality from (3.2) and the convergence of $\sum_{\ell \geq 1} \frac{|b(\ell)|}{\ell^{\alpha}}$.

Let us prove $(i i)$. By assumption, there exist functions $\left(f_{\ell}\right)_{\ell \geq 1}$, such that for every $\ell \geq 1$, $\left(\left(\sum_{1 \leq k \leq n} a(k) f \circ \tau^{\ell k}\right) / A(n)\right)_{n}$ converges $\nu$-a.s. (and in $\left.L^{p}(\nu)\right)$ to $f_{\ell}$. Moreover, $\left\|f_{\ell}\right\|_{p} \leq\|f\|_{p}$. Hence, $g:=\sum_{\ell \geq 1} \frac{b(\ell)}{\ell^{\alpha}} f_{\ell}$ is well-defined in $L^{p}$ and $\nu$-a.s. Let us prove that

$$
\left.\sum_{1 \leq k \leq n} c(k) f \circ \tau^{k}\right) / A(n) \underset{n \rightarrow \infty}{\longrightarrow} g \quad \nu \text {-a.s. }
$$

Let $M \geq 1$ be an integer, fixed for the moment. We have

$$
\begin{aligned}
\sum_{1 \leq k \leq n} c(k) f \circ \tau^{k} & =\sum_{1 \leq \ell \leq n} b(\ell) \sum_{1 \leq k \leq n / \ell} a(k) f \circ \tau^{\ell k} \\
& =\sum_{1 \leq \ell \leq M} b(\ell) \sum_{1 \leq k \leq n / \ell} a(k) f \circ \tau^{\ell k}+\sum_{M<\ell \leq n} b(\ell) \sum_{1 \leq k \leq n / \ell} a(k) f \circ \tau^{\ell k} .
\end{aligned}
$$

Let $g_{M}:=\sum_{\ell \geq M+1} \frac{b(\ell)}{\ell^{\alpha}} f_{\ell}$. We have

$$
\begin{aligned}
\left|\sum_{1 \leq k \leq n} c(k) f \circ \tau^{k} / A(n)-g\right| \leq & \sum_{1 \leq \ell \leq M}\left|\frac{b(\ell)}{\ell^{\alpha}} f_{\ell}-\frac{b(\ell) A(n / \ell)}{A(n)} \frac{1}{A(n / \ell)} \sum_{1 \leq k \leq n / \ell} a(k) f \circ \tau^{\ell k}\right| \\
& +\left|g_{M}\right|+\sum_{M<\ell \leq n} b(\ell) \sum_{1 \leq k \leq n / \ell} a(k) f \circ \tau^{\ell k} .
\end{aligned}
$$

Hence we infer that

$$
\left.\limsup _{n \rightarrow \infty} \mid \sum_{1 \leq k \leq n} c(k) f \circ \tau^{k}\right) / A(n)-g|\leq| g_{M} \mid+C \sum_{\ell>M} \frac{b(\ell)}{\ell^{\alpha}} \mathcal{A}_{\ell}(f) \underset{M \rightarrow \infty}{\longrightarrow} 0 \quad \nu \text {-a.s. }
$$

and the result follows.

Before giving examples, we would like to show that the previous result has a $L^{1, \infty}$ (weak- $L^{1}$ ) version. Recall that $f \in L^{1, \infty}$ if and only if

$$
\|f\|_{1, \infty}:=\sup _{\lambda>0} \lambda \nu(\{x \in X:|f(x)|>\lambda\})<\infty .
$$

The vector space $L_{1, \infty}^{1}$ equipped with $\|\cdot\|_{1, \infty}$ is not a normed-space, but we have the following estimate due to Stein and Weiss [15, Lemma 2.3]. The form stated here is quoted from [9, Lemma 4]. 
Lemma 3.5. Let $\left(g_{n}\right)_{n \in \mathbb{N}}$ be functions in $L^{1, \infty}(X, \mathcal{A}, \nu)$. Assume that

$$
\sum_{n \in \mathbb{N}}\left\|g_{n}\right\|_{1, \infty} \log ^{+}\left(1 /\left\|g_{n}\right\|_{1, \infty}\right)<\infty
$$

Then the series $\sum_{n \in \mathbb{N}} g_{n}$ converges $\nu$-a.s. to an element of $L^{1, \infty}(X, \mathcal{A}, \nu)$. Moreover, writing $L:=\sum_{n \in \mathbb{N}}\left\|g_{n}\right\|_{1, \infty}$ and $K:=\sum_{n \in \mathbb{N}} \frac{\left\|g_{n}\right\|_{1, \infty}}{L} \log \left(L /\left\|g_{n}\right\|_{1, \infty}\right)$, we have,

$$
\left\|\sum_{n \in \mathbb{N}} g_{n}\right\|_{1, \infty} \leq 2(K+2) L \text {. }
$$

We say that $\left(w_{k}\right)_{k \geq 1}$ is a good weight for the dominated ergodic theorem in $L^{1, \infty}$, if there exists $C>0$ such that for every (ergodic) dynamical system $(X, \mathcal{A}, \nu, \tau)$ and every $f$ in $L^{p}$,

$$
\left\|\sup _{n \geq 1} \frac{\left|\sum_{1 \leq k \leq n} w_{k} f \circ \tau^{k}\right|}{W_{n}}\right\|_{1, \infty} \leq C_{p}\|f\|_{1, \infty} .
$$

Proposition 3.6. Let $a(n)$ be a non-negative arithmetic function such that $A(n) \sim n^{\alpha} L(n)$ for some $\alpha>0$ and some non-decreasing slowly varying function $L$. Let $b(n)$ be an arithmetic function such that $\sum_{n \geq 1}|b(n)| / n^{\alpha} \log ^{+}\left(n^{\alpha} / b(n)\right)<\infty, \sum_{n \geq 1} b(n) / n^{\alpha} \neq 0$ and $a * b(n) \geq 0$ for every $n \geq 1$.

(i) Assume that $a(n)$ satisfies to the dominated ergodic theorem in $L^{1, \infty}$. Then, $a * b(n)$ satisfies to the dominated ergodic theorem either.

(ii) If moreover, $a(n)$ satisfies to the pointwise ergodic theorem in $L^{1, \infty}$ then $a * b(n)$ satisfies to the pointwise ergodic theorem either.

Proof. The proof of the maximal inequality follows from (3.3) and Lemma 3.5. Let us prove the pointwise ergodic theorem. As in the proof of Proposition 3.3. (3.4) holds true. Now, the sequence $\left(g_{M}\right)_{M \geq 1}$ from the proof (part $\left.(i i)\right)$ of Proposition 3.3 , converges $\nu$-almost surely to 0 . Moreover, the non-increasing sequence $\left.\sum_{\ell>M} \frac{b(\ell)}{\ell^{\alpha}} \mathcal{A}_{\ell}(f)\right)_{M \geq 1}$ converges $\nu$-a.s. and its limit must be 0 , since, by Lemma 3.5 it converges in probability to 0 .

Theorem 3.7. The arithmetical functions

$$
\begin{cases}\sigma_{s}(k) & \text { the sum of } s \text {-powers of divisors of } k, s \neq 0, \\ \theta(k) & \text { the number of squarefree divisors of } k, \\ J_{s}(k) & \text { the generalized Euler totient function, } s>0, \\ |\mu(k)| & \text { where } \mu \text { is the Möbius function, }\end{cases}
$$

are good weighting functions for the dominated ergodic theorem in $L^{p}, p>1$, and good weighting functions for the pointwise ergodic theorem in $L^{p}, p>1$. Moreover, $\sigma_{s}(s \neq 0),|\mu|$ and $J_{s}(s>0)$ are good weighting functions for the dominated ergodic theorem in $L^{1, \infty}$ and for the pointwise ergodic theorem in $L^{1, \infty}$.

Proof. (i) Denote for $s \in \mathbb{R}$ and all integers $n, \varsigma_{s}(n)=n^{s}$ and let $\mathbb{I}=\varsigma_{0}$. We have $\sigma_{s}=\mathbb{I} * \varsigma_{s}$. If $s<0$, using Birkhoff's Theorem, we see that Proposition 3.3 applies well. Indeed take $a(n)=1$, $b(n)=n^{s}, \alpha=1$. Obviously, $\sum_{n \geq 1} b(n) n^{-1}=\sum_{n \geq 1} n^{-1-|s|}<\infty$ and $\sum_{n \geq 1} b(n) n^{-1} \neq 0$. Thus $\sigma_{s}(n)$ are good weights for the pointwise ergodic theorem in $L^{p}, p \geq 1$ and good weights for the dominated ergodic theorem in $L^{p}, p>1$. If $s>0$, it is well-known (using Abel summation and Birkhoff ergodic theorem) that for any $f \in L^{p}(\nu), p \geq 1, \frac{1}{n^{1+s}} \sum_{k \leq n} k^{s} f \circ \tau^{k} f(x)$ converges almost everywhere as $n \rightarrow \infty$. We apply Proposition 3.3 with $a(n)=n^{s}, b(n)=1, \alpha=1+s$. This shows that $\sigma_{s}(n)$ are good weights for the pointwise ergodic theorem in $L^{p}, p>1$. They are also good weights for the dominated ergodic theorem in $L^{p}, p>1$, since $\frac{1}{n^{1+s}}\left|\sum_{k \leq n} k^{s} f \circ \tau^{k} f(x)\right| \leq$ $\frac{1}{n} \sum_{k \leq n}\left|f \circ \tau^{k} f(x)\right|$.

(ii) Let us now consider the arithmetical function $\theta$. Introduce the arithmetical functions

$$
\delta(n)=\left\{\begin{array}{ll}
1 & n=1, \\
0 & \text { unless. }
\end{array} \quad \tilde{\mu}(n)= \begin{cases}\mu(d) & n=d^{2}, \\
0 & \text { unless } .\end{cases}\right.
$$


Recall the fundamental inversion formula $\delta=\mathbb{I} * \mu$. Writing $n=q m^{2}$, where $q$ is the product of those prime factors of $n$ with odd exponents, we first notice that

$$
\mu(n)^{2}=\mu\left(m^{2}\right)^{2}=\delta(m)=\mathbb{I} * \mu(m)=\sum_{d^{2} \mid n} \mu(d)=\sum_{u \mid n} \tilde{\mu}(u)=\mathbb{I} * \tilde{\mu}(n)
$$

since $d \mid m$ if and only if $d^{2} \mid n$. Now as $d=\mathbb{I} * \mathbb{I}$,

$$
\theta(n)=\sum_{d \mid n}|\mu(d)|=\sum_{d \mid n} \mu(d)^{2}=\sum_{d \mid n} \mathbb{I} * \tilde{\mu}(d)=\mathbb{I} * \mathbb{I} * \tilde{\mu}(n)=d * \tilde{\mu}(n) .
$$

Moreover, $\sum_{n \geq 1} \frac{|\tilde{\mu}(n)|}{n} \leq \sum_{n \geq 1} \frac{1}{n^{2}}<\infty$ and $\sum_{n \geq 1} \frac{\tilde{\mu}(n)}{n}=\sum_{n \geq 1} \frac{\mu(n)}{n^{2}}=\frac{1}{\zeta(2)} \neq 0$. The conclusion thus follows from Proposition 3.3 and Theorem 1.1

(iii) Recall that $J_{s}(n)=\varsigma_{s} * \mu(n)=\sum_{d \mid n} d^{s} \mu\left(\frac{n}{d}\right)$. The proof is very similar to the one of the case $\sigma_{s}(n)=n^{s}, s>0$. We apply Proposition 3.3 with $a(n)=n^{s}, b(n)=\mu(n), \alpha=1+s$, noticing that $\sum_{n \geq 1} \frac{\mu(n)}{n^{1+s}}=\frac{1}{\zeta(1+s)} \neq 0$.

(iv) This follows from Birkhoff's theorem since $\mu(n)^{2}=\mathbb{I} * \tilde{\mu}(n)$ and $\sum_{n \geq 1} \frac{|\tilde{\mu}(n)|}{n}<\infty$

\section{Sketch of Bourgain's approach.}

Before passing to the preparation of the proof of Theorem 1.2, it is necessary to briefly recall the essential steps of Bourgain's method. We refer ourselves to [5]. The basic reduction (Calderon's transference principle) to the shift model $(\mathbb{Z}, S)$, where $S \underline{z}=\left\{z_{\ell+1}, \ell \in \mathbb{Z}\right\}, \underline{z}=\left\{z_{\ell}, \ell \in \mathbb{Z}\right\}$ can be presented as follows. Let $(X, \alpha, \mu, \tau)$ be a measurable dynamical system and let $1<p \leq \infty$. Let $J, N$ be positive integers with $J \gg N$. Let $f \in L^{p}(\mu), x \in X$ and define $\varphi$ on $\mathbb{Z}$ by

$$
\varphi(j)=\left\{\begin{array}{lc}
f\left(\tau^{j} x\right) & \text { if } 0 \leq j \leq J \\
0 & \text { otherwise }
\end{array}\right.
$$

We note that

$$
A_{n}^{\tau} f\left(\tau^{j} x\right)=\frac{1}{W_{n}} \sum_{k=0}^{n-1} w_{k}\left(S^{k} \varphi\right)(j), \quad n \leq N, 0 \leq j<J-N .
$$

Hence

$$
\sum_{0 \leq j<J-N} \sup _{n=1}^{N}\left|A_{n}^{\tau} f\left(\tau^{j} x\right)\right| \leq \sum_{0 \leq j<J-N} \sup _{n=1}^{N}\left|\frac{1}{W_{n}} \sum_{k=0}^{n-1} w_{k} S^{k} \varphi(j)\right| .
$$

Assume that we have proved that

$$
\left\|\sup _{n \geq 1}\left|\frac{1}{W_{n}} \sum_{k=0}^{n-1} w_{k} S^{k} g(j)\right|\right\|_{\ell^{p}(\mathbb{Z}, d j)} \leq C_{p}\|g\|_{\ell^{p}(\mathbb{Z}, d j)},
$$

for any $g \in \ell^{p}(\mathbb{Z})$. Taking $g=\varphi$ we deduce,

$$
\sum_{0 \leq j<J-N} \sup _{n=1}^{N}\left|A_{n}^{\tau} f\left(\tau^{j} x\right)\right|^{p} \leq C_{p}^{p} \sum_{0 \leq j \leq J}\left|f\left(\tau^{j} x\right)\right|^{p} .
$$

By integrating with respect to $\mu$, it follows that

$$
\sum_{0 \leq j<J-N}\left\|\sup _{n=1}^{N}\left|A_{n}^{\tau} f \circ \tau^{j}\right|\right\|_{p}^{p} \leq C_{p}^{p} \sum_{0 \leq j \leq J}\left\|f \circ \tau^{j}\right\|_{p}^{p} .
$$

Since $\tau$ is $\mu$-preserving, this finally leads to

$$
\left\|\sup _{n \geq 1}\left|A_{n}^{\tau} f\right|\right\|_{p} \leq C(p)\|f\|_{p}
$$

Consider the kernel $K_{n}: \ell^{p}(\mathbb{Z}) \rightarrow \ell^{p}(\mathbb{Z})$ defined by

$$
K_{n}=\frac{1}{W_{n}} \sum_{k=0}^{n-1} w_{k} \delta_{\{k\}} .
$$


By Fourier inversion formula, the maximal inequality on the shift model

$$
\left\|\sup _{n \in \mathbb{N}}\left|K_{n} * f\right|\right\|_{p} \leq C\|f\|_{p}
$$

is equivalent to

$$
\left\|\sup _{n \in \mathbb{N}}\left|\int_{0}^{1} \bar{K}_{n}(t) \widehat{f}(t) \mathrm{e}^{2 i \pi j t} d t\right|\right\|_{\ell^{p}(\mathbb{Z}, d j)}<\infty .
$$

The latter is obtained by first proving a maximal inequality relatively to another kernel $L_{n}$, whose Fourier transform is close to that of $K_{n}$, by using Fourier analysis, and next establishing an approximation result of the type

$$
\left\|\widehat{K}_{n}-\widehat{L}_{n}\right\|_{\infty} \leq \frac{C}{(\log n)^{b}} \quad \forall n \geq 2
$$

where $b$ is some positive constant. In several situations (in particular, when $w_{n}=d_{n}$ ), in order to deduce the maximal inequality for $K_{n}$, there is no loss to assume that $f \geq 0$ and to restrict $n$ to dyadic values $\left(n=2^{k}, k \in \mathbb{N}\right)$. The plain inequality

$$
\sup _{k \in \mathbb{N}}\left|f * K_{2^{k}}\right| \leq \sup _{k \in \mathbb{N}}\left|f * L_{2^{k}}\right|+\left(\sum_{k \in \mathbb{N}}\left|f *\left(K_{2^{k}}-L_{2^{k}}\right)\right|^{2}\right)^{1 / 2}
$$

implies since $\| f *\left(K_{2^{k}}-L_{2^{k}}\left\|_{2} \leq\right\| K_{2^{k}}-L_{2^{k}}\left\|_{\infty}\right\| f \|_{2}\right.$,

$$
\left\|\sup _{k \in \mathbb{N}}\left|f * K_{2^{k}}\right|\right\|_{2} \leq\left\|\sup _{k \in \mathbb{N}}\left|f * L_{2^{k}}\right|\right\|_{2}+\left(\sum_{k \in \mathbb{N}}\left\|K_{2^{k}}-L_{2^{k}}\right\|_{\infty}^{2}\right)^{1 / 2}\|f\|_{2} .
$$

Now let $\rho>1$ and denote $I_{\rho}:=\left\{\left[\rho^{n}\right]: n \in \mathbb{N}\right\}$. The convergence almost everywhere will result from the inequality: for every $\rho>1$ and every sequence $\left(N_{j}\right)_{j \geq 1}$, with $N_{j+1} \geq 2 N_{j}$,

$$
\sum_{1 \leq j \leq J}\left\|\sup _{\substack{N_{j} \leq N \leq N_{j+1} \\ N \in I_{\rho}}}\left|A_{n} f-A_{N_{j}} f\right|\right\|_{2} \leq o(J)\|f\|_{2},
$$

for $J$ large depending on $\rho$. Consequently, once the reduction to the shift model operated, the main steps in applying Bourgain's approach are summarized in (4.2) and (4.3), see (7.12) and Theorem 7.5. The next sections are devoted to the necessary preparatory steps for the application of this method.

Remark 4.1 (Maximal shift inequality). For the Cesáro kernel $\kappa_{n}=\frac{1}{n} \sum_{k=0}^{n-1} \delta_{\{k\}}$, the maximal shift inequality for $p>1$ writes (after variable change),

$$
\sum_{i \in \mathbb{Z}} \sup _{j \geq i} \frac{1}{j-i+1}\left|\sum_{l=i}^{j} g(l)\right|^{p} \leq C_{p}^{p} \sum_{i \in \mathbb{Z}}|g(i)|^{p} .
$$

It suffices to prove it for $g \geq 0$. Assume first that $\operatorname{support}(g)=\mathbb{Z}_{-}$. Then the only sums playing a role are those with $i \leq j \leq-1$ and the left-term writes

$$
\sum_{\delta \geq 1} \sup _{1 \leq \gamma \leq \delta}\left(\frac{1}{\delta-\gamma+1} \sum_{u=\gamma}^{\delta} g(-u)\right)^{p}
$$

Applying Hardy and Littlewood maximal inequality ([10], Theorem 8),

$$
\sum_{j=1}^{\infty} \max _{1 \leq i \leq j}\left(\frac{1}{j-i+1} \sum_{l=i}^{j} a_{l}\right)^{p}<\left(\frac{p}{p-1}\right)^{p} \sum_{n=1}^{\infty} a_{n}^{p} \quad\left(a_{n} \geq 0\right),
$$

shows that (4.4) is realized with $C_{p}=p /(p-1)$. Now if $\operatorname{support}(g)=(-\infty, M]$, we apply the previous estimate to $\widetilde{g}(k)=g(k+M)$ whose support is $\mathbb{Z}_{-}$. To pass to the general case, we use monotone convergence theorem (letting $M$ tend to $+\infty$ ), which is justified since $g \geq 0$. 


\section{Divisors estimates.}

Recall that the divisor function is defined by $d(n):=\#\{1 \leq d \leq n: d \mid n\}$. For every $x \in[0,1]$, define

$$
D_{n}(x):=\sum_{1 \leq k \leq n} d(k) \mathrm{e}^{2 i k \pi x}
$$

Then

$$
\begin{aligned}
D_{n}(x) & =\sum_{1 \leq k \ell \leq n} \mathrm{e}^{2 i k \ell \pi x}=2 \sum_{1 \leq k \leq \sqrt{n}} \sum_{1 \leq \ell \leq n / k} \mathrm{e}^{2 i k \ell \pi x}-\sum_{1 \leq k, \ell \leq \sqrt{n}} \mathrm{e}^{2 i k \ell \pi x} \\
& :=\widetilde{D}_{n}(x)-\sum_{1 \leq k, \ell \leq \sqrt{n}} \mathrm{e}^{2 i k \ell \pi x}
\end{aligned}
$$

It is well-known that

$$
\left.D_{n}:=D_{n}(0)=n(\log n+2 \gamma-1)+O\left(n^{1 / 3}\right)\right)
$$

where $\gamma$ is the Euler constant. Better estimates of the error term exist, but we shall not need them. Several asymptotics for $\left(D_{n}(x)\right)_{n}$ may be found in Jutila [12] when $x$ is rational or in Wilton [19] for general $x$ under conditions on the continuous fractions expansion of $x$.

We shall need quantitative asymptotics according to the fact that $x$ is close to rational numbers with small or large denominators. In particular, it is unclear how to derive the results that we need from the above mentionned papers.

Our estimates use very simple ideas and we do not make use of the Voronoi identity related to the problem. Actually, we shall rather estimate $\widetilde{D}_{n}(x)$. We note throughout by $a \wedge b$ the greatest common divisor of the positive integers $a$ and $b$.

Lemma 5.1. There exists $C>0$, such that for every $1 \leq a \leq q$ with $a \wedge q=1$, or $a=0, q=1$, and every $n \geq 1$, we have

$$
\left.\left|D_{n}(a / q)-\frac{n}{q}(\log n-2 \log q+2 \gamma-1)\right| \leq C(\sqrt{n}+q) \log (q+1)\right) .
$$

Proof. The case $a=0, q=1$ follows from (5.1).

1. Assume first that $q \leq \sqrt{n}$. We split the sum defining $\widetilde{D}_{n}$ according to the fact that $k$ is a multiple of $q$ or not. We use the following obvious facts.

- If $q \mid k$, we have

$$
\sum_{1 \leq \ell \leq n / k} \mathrm{e}^{2 i k \ell \pi a / q}=[n / k] .
$$

- If there exists $1 \leq s \leq q-1$, such that $k \equiv s \bmod q$, we have

$$
\left|\sum_{1 \leq \ell \leq n / k} \mathrm{e}^{2 i k \ell \pi a / q}\right| \leq \frac{2}{\left|1-\mathrm{e}^{2 i s \pi a / q}\right|}
$$

Now, there are $[\sqrt{n} / q]$ multiple of $q$ less than $\sqrt{n}$ and for every $1 \leq s \leq q-1$, there are at most $[\sqrt{n} / q]$ integers smaller than $\sqrt{n}$ and congruent to $s \bmod q$.

Notice that $s \rightarrow a s$ is a bijection of $\mathbb{Z} / q \mathbb{Z}-\{0\}$ and that there exists $C>0$, such that for every $1 \leq s^{\prime} \leq q-1$,

$$
\frac{2}{\left|1-\mathrm{e}^{2 i \pi s^{\prime} / q}\right|} \leq \frac{C q}{\min \left(s^{\prime}, q-s^{\prime}\right)} .
$$

Hence, writing $\Gamma_{n}:=\{1 \leq k \leq \sqrt{n}: k \notin q \mathbb{Z}\}$,

$$
\left|\sum_{k \in \Gamma_{n}} \sum_{1 \leq \ell \leq n / k} \mathrm{e}^{2 i k \ell \pi a / q}\right| \leq[\sqrt{n} / q] \sum_{1 \leq s \leq q / 2} \frac{C q}{s} \leq \widetilde{C} \sqrt{n} \log (q+1) .
$$


Recall (see for instance Tenenbaum [16] page 6) that there exists a universal constant $C>0$, such that for every $n \geq 1$,

$$
\left|\sum_{1 \leq m \leq n} \frac{1}{m}-\log n-\gamma\right| \leq \frac{C}{n}
$$

where $\gamma$ is Euler's constant.

Then, using that $\mid \log \left(\frac{\sqrt{n}}{q}\right)-\log \left(\left[\frac{\sqrt{n}}{q}\right]\right) \leq \frac{2 q}{\sqrt{n}}$, we infer that,

$$
\begin{aligned}
\widetilde{D}_{n}(a / q) & =2 \sum_{1 \leq m \leq[\sqrt{n} / q]} n /(m q)+\mathcal{O}(\sqrt{n} \log (q+1)) \\
& =\frac{n}{q}(\log n-2 \log q+2 \gamma)+\mathcal{O}(\sqrt{n})+\mathcal{O}(\sqrt{n} \log (q+1)),
\end{aligned}
$$

where the "big $\mathcal{O}$ " are uniform in the parameters.

Similar computations give,

$$
\sum_{1 \leq k, \ell \leq \sqrt{n}} \mathrm{e}^{2 i \pi k \ell \pi \frac{a}{q}}=\frac{n}{q}+O(\sqrt{n} \log (q+1)) .
$$

2. Assume now that $q>\sqrt{n}$. We use a similar reasonning as above. In that case no integer $k$, $1 \leq k \leq \sqrt{n}$, is a multiple of $q$ and $\{a k: 1 \leq k \leq \sqrt{n}\}$ is a set of integers with distinct residues modulo $q$.

Hence,

$$
\left|D_{n}(a / q)\right| \leq \sum_{1 \leq k \leq \sqrt{n}} \frac{2}{\mid 1-\mathrm{e}^{2 i \pi k a / q \mid}} \leq C \sum_{1 \leq|\ell| \leq q / 2} \frac{2 q}{|\ell|} \leq C q \log (q+1) .
$$

Similarly,

$$
\left|\sum_{1 \leq k, \ell \leq \sqrt{n}} \mathrm{e}^{2 i k \ell \pi \frac{a}{q}}\right| \leq C q \log (q+1) .
$$

Now, since $q>\sqrt{n}$, we see that $\frac{n}{q}|\log n-2 \log q+2 \gamma-1| \leq C q \log (q+1)$, and the lemma is proved.

Now let $\left(P_{n}\right)_{n \geq 1}$ and $\left(Q_{n}\right)_{n \geq 1}$ be non-decreasing sequences of integers, such that for every $n \geq 1,1 \leq P_{n} \leq \bar{Q}_{n} \leq n$.

Lemma 5.2. Let $1 \leq a \leq q \leq P_{n}$ with $a \wedge q=1$, or $a=0, q=1$. Let $x \in[0,1]$ be such that $|x-a / q| \leq 1 / Q_{n}$. There exists some universal constant $C>0$ such that, for every $n \geq 1$,

$$
\begin{gathered}
\left|D_{n}(x)-\frac{1}{q} \sum_{1 \leq k \leq n} \log k \mathrm{e}^{2 i k \pi(x-a / q)}-\frac{2(\gamma-1-\log q)}{q} \sum_{1 \leq k \leq n} \mathrm{e}^{2 i \pi k(x-a / q)}\right| \\
\leq C\left(\frac{n^{3 / 2} \log n}{Q_{n}}+\frac{n P_{n} \log n}{Q_{n}}\right) .
\end{gathered}
$$

In particular, there exists $\widetilde{C}>0$, such that, for every $n \geq 1$,

$$
\left|D_{n}(x)-\frac{\log n}{q} \sum_{1 \leq k \leq n} \mathrm{e}^{2 i k \pi(x-a / q)}\right| \leq \widetilde{C}\left(n+\frac{n^{3 / 2} \log n}{Q_{n}}+\frac{n P_{n} \log n}{Q_{n}}\right) .
$$

Remark 5.3. The simpler estimate (5.5) will allow us to prove the oscillation inequality in $L^{2}(\mu)$. If $K_{n}=\frac{1}{D_{n}} \sum_{1 \leq k \leq n} d(k) \delta_{\{k\}}$ and $k_{n}=\frac{\log n}{D_{n}} \sum_{1 \leq k \leq n} \delta_{\{k\}}$, it will provide (upon suitable choice of $\left.P_{n}, Q_{n}\right)$ the estimate

$$
\left|K_{n}(x)-\frac{1}{q} k_{n}(x-a / q)\right| \leq \frac{C}{\log n} .
$$

It is also sufficient to prove the maximal inequality in $L^{p}(\mu)$ for $3 / 2<p \leq 2$. However, (5.4) seems to be needed to prove the maximal inequality for $1<p \leq 2$. 
Proof. We have, writing $R_{n}:=\frac{n}{q}(\log n-2 \log q+2 \gamma-1)$ and $R_{0}=0$,

$$
\begin{aligned}
D_{n}(x)= & \sum_{1 \leq k \leq n} d(k) \mathrm{e}^{2 i \pi k x}=\sum_{1 \leq k \leq n} d(k) \mathrm{e}^{2 i \pi k a / q} \mathrm{e}^{2 i \pi k(x-a / q)} \\
= & \sum_{1 \leq k \leq n}\left(d(k) \mathrm{e}^{2 i \pi k a / q}-\left(R_{k}-R_{k-1}\right)\right) \mathrm{e}^{i k(x-a / q)} \\
& \quad+\sum_{1 \leq k \leq n}\left(R_{k}-R_{k-1}\right) \mathrm{e}^{2 i \pi k(x-a / q)} \\
& :=T_{n}+U_{n} .
\end{aligned}
$$

Notice that

$$
\begin{aligned}
q\left(R_{k}-R_{k-1}\right) & =k \log k-(k-1) \log (k-1)-2 \log q+2 \gamma-1 \\
& =\log k-2 \log q+2 \gamma-2+\mathcal{O}(1 / k)
\end{aligned}
$$

Hence,

$$
q U_{n}=\sum_{1 \leq k \leq n} \log k \mathrm{e}^{2 i \pi k(x-a / q)}+2(\gamma-1-\log q) \sum_{1 \leq k \leq n} \mathrm{e}^{2 i \pi k(x-a / q)}+O(\log n) .
$$

To deal with $T_{n}$ we use Abel summation by part. Recall that by Lemma 5.1, for every $1 \leq k \leq n$, $\left|D_{k}(a / q)-R_{k}\right| \leq C \sqrt{k}(\log k+\log (q+1))$. We have

$$
\begin{gathered}
T_{n}=\sum_{1 \leq k \leq n}\left(\left(D_{k}(a / q)-R_{k}\right)-\left(D_{k-1}(a / q)-R_{k-1}\right)\right) \mathrm{e}^{2 i \pi k(x-a / q)} \\
=\sum_{1 \leq k \leq n}\left(D_{k}(a / q)-R_{k}\right) \mathrm{e}^{2 i \pi k(x-a / q)}\left(1-\mathrm{e}^{2 i \pi(x-a / q)}\right)+\left(D_{n}(a / q)-R_{n}\right) \mathrm{e}^{2 i \pi(n+1)(x-a / q)} .
\end{gathered}
$$

Hence,

$$
\left|T_{n}\right| \leq\left|D_{n}(a / q)-R_{n}\right|+\frac{C}{Q_{n}} \sum_{1 \leq k \leq n}\left|D_{k}(a / q)-R_{k}\right| \leq C \frac{n^{3 / 2} \log n}{Q_{n}} .
$$

Let us prove (5.5). Clearly, it suffices to handle the first term in (5.4). We have

$$
\left|\sum_{1 \leq k \leq n} \log k \mathrm{e}^{2 i \pi k(x-a / q)}-\log n \sum_{1 \leq k \leq n} \mathrm{e}^{2 i \pi k(x-a / q)}\right| \leq \sum_{1 \leq k \leq n}|\log (k / n)| \leq n \int_{0}^{1}|\log t| d t,
$$

which finishes the proof.

Lemma 5.4. Let $x \in[0,1]$ be such that for every $1 \leq q \leq P_{n}$ and every $0 \leq a \leq q,|x-a / q|>$ $1 / Q_{n}$. There exists some absolute constant $C>0$ such that

$$
\left|D_{n}(x)\right| \leq C\left(\frac{n \log n}{P_{n}}+\sqrt{n} \log n+Q_{n} \log n+\frac{n^{2} \log n}{P_{n} Q_{n}}\right) .
$$

Proof. By the Dirichlet principle, there exists $1 \leq a \leq q$ with $a \wedge q=1$, such that $|x-a / q| \leq$ $1 /\left(q Q_{n}\right) \leq 1 / Q_{n}$. By assumption, we must have $q>P_{n}$, hence we have

$$
|x-a / q| \leq \frac{1}{P_{n} Q_{n}} .
$$

Then, using that $\left|\mathrm{e}^{2 i p k x}-\mathrm{e}^{2 i \pi k a / q}\right| \leq\left|1-\mathrm{e}^{2 i \pi k(x-a / q)}\right| \leq 2 \pi k|x-a / q| \leq 2 \pi k /\left(P_{n} Q_{n}\right)$, we infer that

$$
\left|D_{n}(x)-D_{n}(a / q)\right| \leq \frac{2 \pi}{P_{n} Q_{n}} \sum_{1 \leq k \leq n} k d(k) \leq \widetilde{C} \frac{n^{2} \log n}{P_{n} Q_{n}} .
$$

To conclude, we use Lemma 5.1, noticing that $q \geq P_{n}$. 


\section{Maximal inequalities in $\ell^{p}$}

In this section we recall some results of Fourier analysis that may be found in [18], see also [4] or [5] for related results.

In all that section, we denote by $\eta: \mathbb{R} \rightarrow[0,1]$ a (fixed) smooth function such that

$$
\eta(x)= \begin{cases}1 & \text { if } x \in\left[-\frac{1}{4}, \frac{1}{4}\right] \\ 0 & \text { if } x \in \mathbb{R} \backslash[-1 / 2,1 / 2] \\ \text { is } C^{\infty} & \text { on }[-1 / 2,1 / 2] \backslash\left[-\frac{1}{4}, \frac{1}{4}\right]\end{cases}
$$

Further, $\left(w_{n}\right)_{n \geq 1}$ will be a sequence of elements of $\ell^{1}(\mathbb{Z})$ such that for every $p>1$, there exists $C_{p}(\omega)>0$ such that

$$
\left\|\sup _{n \geq 1}\left|w_{n} * g\right|\right\|_{\ell^{p}(\mathbb{Z})} \leq C_{p}(w)\|g\|_{\ell^{p}(\mathbb{Z})} \quad \forall g \in \ell^{p}(\mathbb{Z}) .
$$

We follow here the approach of Wierdl [18. However, as it has been noticed very recently by Mirek and Trojan [13, there is a small gap in Wierdl's argument (on should have $q^{p}$ instead of $q$ in the equation after $* *$ page 331 ), hence we shall sketch some of the proofs. Our first lemma is just equation (24) of Wierdl [18, which is independent from the gap.

Lemma 6.1. There exists $M>2$ (depending solely on $\eta$ ) such that for every $p>1$, there exists $C_{p}>0$ such that for every $Q>1$ and every $1 \leq d \leq Q / M$ and every $h \in \ell^{p}(\mathbb{Z})$ we have

$$
\left\|\sup _{n}\left|\int_{-1 / 2}^{1 / 2} \widehat{w}_{n}(x) \eta(Q x) \widehat{h}(x) \mathrm{e}^{2 i \pi d j x} d x\right|\right\|_{\ell^{p}(\mathbb{Z}, d j)} \leq \frac{C_{p} C_{p}(w)}{d^{1 / p}}\|h\|_{\ell^{p}},
$$

Our second lemma is the correct version of Lemma $3^{\prime}$ of Wierdl [18. The term $d^{1-1 / p}$ does not appear in Lemma $3^{\prime}$. Since we shall apply Lemma 6.2 for $p$ close to 1 , it will turn out that this extra term will not be disturbing.

Lemma 6.2. There exists $M>2$ (depending solely on $\eta$ ) such that for every $p>1$, there exists $C_{p}>0$ such that for every $Q>1$, every $g \in \ell^{p}(\mathbb{Z})$,

$$
\left\|\sup _{n}\left|\sum_{1 \leq m \leq d} \int_{-1 / 2}^{1 / 2} \widehat{w}_{n}(x) \eta(Q x) \widehat{g}(m / d+x) \mathrm{e}^{2 i \pi j(m / d+x)} d x\right|\right\|_{\ell^{p}(\mathbb{Z}, d j)} \leq C_{p} C_{p}(w) d^{1-1 / p}\|g\|_{\ell^{p}},
$$

whenever $1 \leq d \leq Q / M$.

Proof. We proceed as in Wierdl [18. We first assume that $g$ has finite support, i.e. there exists $N>0$, such that $g(k)=0$ whenever $|k|>N$. We have $\widehat{g}(m / d+x)=\sum_{k=-N}^{N} g(k) \mathrm{e}^{2 i k \pi(m / d+x)}$. Notice that $\sum_{1 \leq m \leq d} \mathrm{e}^{2 i \pi(k+j) m / d}=d$ if $d \mid(k+j)$ and 0 otherwise. Hence, for every $x \in[1 / 2,1 / 2]$ and every $j \in \mathbb{Z}$, writing $j=t d+r$ with $1 \leq t \leq d$, we have

$$
\begin{aligned}
\sum_{1 \leq m \leq d} \widehat{g}(m / d+x) \mathrm{e}^{2 i \pi j(m / d+x)} & =\sum_{k=-N}^{N} g(k) \mathrm{e}^{2 i \pi(k+j) x} \sum_{1 \leq m \leq d} \mathrm{e}^{2 i \pi(k+j) m / d} \\
& =d \sum_{s \in \mathbb{Z}} g(s d-j) \mathrm{e}^{2 i \pi s d x} \\
& =d \mathrm{e}^{2 i \pi t d x} \sum_{s \in \mathbb{Z}} g(s d-r) \mathrm{e}^{2 i \pi s d x}
\end{aligned}
$$

Define $\widehat{h}_{d, r} \in \ell^{p}(\mathbb{Z})$ (with finite support) by its Fourier transform:

$$
\widehat{h}_{d, r}(x):=d \sum_{s \in \mathbb{Z}} g(s d-r) \mathrm{e}^{2 i \pi s d x} .
$$

Then, using Lemma 6.1, we infer that

$$
\left\|\sup _{n}\left|\sum_{1 \leq m \leq d} \int_{-1 / 2}^{1 / 2} \widehat{w}_{n}(x) \eta(Q x) \widehat{g}(m / d+x) \mathrm{e}^{2 i \pi j(m / d+x)} d x\right|\right\|_{\ell^{p}(\mathbb{Z}, d j)}^{p}
$$




$$
\begin{aligned}
& =\sum_{1 \leq r \leq d}\left\|\sup _{n}\left|\int_{-1 / 2}^{1 / 2} \widehat{w}_{n}(x) \eta(Q x) \widehat{h}_{d, r}(x) \mathrm{e}^{2 i \pi d t x} d x\right|\right\|_{\ell^{p}(\mathbb{Z}, d t)}^{p} \\
& \leq \frac{C_{p} C_{p}(w)}{d} \sum_{1 \leq r \leq d}\left\|h_{d, r}\right\|_{\ell^{p}(\mathbb{Z})}^{p} .
\end{aligned}
$$

By construction, $\left\|h_{d, r}\right\|_{\ell^{p}(\mathbb{Z})}^{p}=d^{p} \sum_{s \in \mathbb{Z}} g(s d-r)^{p}$. Hence, $\sum_{1 \leq r \leq d}\left\|h_{d, r}\right\|_{\ell^{p}(\mathbb{Z})}^{p}=d^{p}\|g\|_{\ell^{p}(\mathbb{Z})}^{p}$, and the result follows. The case where $g$ has no finite support may be deduced by approximation.

Lemma 6.3. There exists $M>2$ (depending solely on $\eta$ ) and $C>0$ such that for every $Q>1$, every $g \in \ell^{2}(\mathbb{Z})$,

$$
\left\|\sup _{n}\left|\sum_{1 \leq m \leq d} \int_{-1 / 2}^{1 / 2} \widehat{w}_{n}(x) \eta(Q x) \widehat{g}(m / d+x) \mathrm{e}^{2 i \pi j(m / d+x)} d x\right|\right\|_{\ell^{2}(\mathbb{Z}, d j)} \leq C C_{2}(w)\|g\|_{\ell^{2}},
$$

whenever $1 \leq d \leq Q / M$.

Proof. We have

$$
\begin{aligned}
\Delta & :=\left\|\sup _{n}\left|\sum_{1 \leq m \leq d} \int_{-1 / 2}^{1 / 2} \widehat{w}_{n}(x) \eta(Q x) \widehat{g}(m / d+x) \mathrm{e}^{2 i \pi j(m / d+x)} d x\right|\right\|_{\ell^{2}(\mathbb{Z}, d j)}^{2} \\
& =\sum_{j \in \mathbb{Z}} \sup _{n}\left|\sum_{1 \leq m \leq d} \int_{-1 / 2}^{1 / 2} \widehat{w}_{n}(x) \eta(Q x) \widehat{g}(m / d+x) \mathrm{e}^{2 i \pi j(m / d+x)} d x\right|^{2} .
\end{aligned}
$$

For $1 \leq r \leq d$, define $g_{r}$ by

$$
\widehat{g}_{r}(x)=\sum_{1 \leq m \leq d} \widehat{g}(m / d+x) \mathrm{e}^{2 i \pi r(m / d+x)} .
$$

Splitting the previous series into $d$ series according with the residue class of $j$ mod $d$ we see that

$$
\Delta=\sum_{1 \leq r \leq d} \sum_{j \in \mathbb{Z}} \sup _{n}\left|\int_{-1 / 2}^{1 / 2} \widehat{w}_{n}(x) \eta(Q x) \widehat{g}_{r}(x) \mathrm{e}^{2 i \pi j d x} d x\right|^{2}
$$

Notice that $\eta\left(\frac{Q}{2} \cdot\right) \eta(Q \cdot)=\eta(Q \cdot)$. By Lemma 6.1 applied with $\widehat{h}(x)=\eta\left(\frac{Q}{2} x\right) \widehat{g}_{r}(x)$, we have, by Parseval

$$
\sum_{j \in \mathbb{Z}} \sup _{n}\left|\int_{-1 / 2}^{1 / 2} \widehat{w}_{n}(x) \eta(Q x) \widehat{g}_{r}(x) \mathrm{e}^{2 i \pi j d x} d x\right|^{2} \leq \frac{C_{2}}{d} \int_{-1 / 2}^{1 / 2}\left|\eta\left(\frac{Q}{2} x\right) \widehat{g}_{r}(x)\right|^{2} d x .
$$

Now,

$$
\left|\eta\left(\frac{Q}{2} x\right) \widehat{g}_{r}(x)\right|^{2}=\eta^{2}\left(\frac{Q}{2} x\right) \sum_{1 \leq m, m^{\prime} \leq d} \widehat{g}(m / d+x) \overline{\widehat{g}\left(m^{\prime} / d+x\right)} \mathrm{e}^{2 i \pi r\left(m-m^{\prime}\right) / d} .
$$

Hence, using that $\sum_{1 \leq r \leq d} \mathrm{e}^{2 i \pi r\left(m-m^{\prime}\right) / d}$ is equal to 0 if $m \neq m^{\prime}$ and to $d$ if $m=m^{\prime}$, we obtain that

$$
\begin{aligned}
\sum_{1 \leq r \leq d}\left|\eta\left(\frac{Q}{2} x\right) \widehat{g}_{r}(x)\right|^{2} & =\eta^{2}\left(\frac{Q}{2} x\right) \sum_{1 \leq m, m^{\prime} \leq d} \widehat{g}(m / d+x) \overline{\widehat{g}\left(m^{\prime} / d+x\right)} \sum_{1 \leq r \leq d} \mathrm{e}^{2 i \pi r\left(m-m^{\prime}\right) / d} \\
& =\eta^{2}\left(\frac{Q}{2} x\right) \sum_{1 \leq m \leq d}|\widehat{g}(m / d+x)|^{2}
\end{aligned}
$$

Then, we infer that,

$$
\begin{aligned}
\Delta & \leq C_{2}^{2} \sum_{1 \leq m \leq d} \int_{-1 / 2}^{1 / 2}\left(\eta\left(\frac{Q}{2} x\right)\right)^{2}|\widehat{g}(m / d+x)|^{2} d x \\
& =C_{2}^{2} \sum_{1 \leq m \leq d} \int_{-1 / 2}^{1 / 2}\left(\eta\left(\frac{Q}{2}(x-m / d)\right)\right)^{2}|\widehat{g}(x)|^{2} d x .
\end{aligned}
$$


But, if $M>2$, the functions $\left(\eta\left(\frac{Q}{2}(\cdot-m / d)\right)\right)_{1 \leq d \leq Q / m}$ have disjoint supports. Hence,

$$
\Delta \leq C_{2}^{2}\|g\|_{\ell^{2}}^{2}
$$

and the proof is complete.

For every $s \geq 0$, define $\eta_{s}$ by

$$
\eta_{s}(x)=\eta\left(4^{s} M x\right)
$$

where $M$ is a constant such that Lemma 6.1 and Lemma 6.3 apply.

Corollary 6.4. Let $p>1$. For every $\varepsilon>0$, there exists $C_{p}>0$ such that for every $s \geq 1$ and every $1 \leq q<4^{s}$ and every $g \in \ell^{p}(\mathbb{Z})$,

$\left\|\sup _{n}\left|\sum_{1 \leq a \leq q, a \wedge q=1} \int_{-1 / 2}^{1 / 2} \widehat{w}_{n}(x) \eta_{s}(x) \widehat{g}(a / q+x) \mathrm{e}^{2 i \pi j(a / q+x)} d x\right|\right\|_{\ell^{p}(\mathbb{Z}, d j)} \leq C_{p} C_{p}(w) q^{1+\varepsilon-1 / p}\|g\|_{\ell^{p}}$

If $p=2$,

$$
\left\|\sup _{n} \mid \sum_{1 \leq a \leq q, a \wedge q=1} \int_{-1 / 2}^{1 / 2} \widehat{w}_{n}(x) \eta_{s}(x) \widehat{g}(a / q+x) \mathrm{e}^{2 i \pi j(a / q+x)} d x\right\|_{\ell^{2}(\mathbb{Z}, d j)} \leq C_{2} C_{2}(w) q^{\varepsilon}\|g\|_{\ell^{2}} .
$$

Proof. As we have for any function on $\mathbb{R}, \sum_{k=1}^{n} F\left(\frac{k}{n}\right)=\sum_{q \mid n} \sum_{\substack{1 \leq a \leq q \\ a \wedge q=1}} F\left(\frac{a}{q}\right)$, it follows from Möbius inversion formula that

$$
\sum_{1 \leq a \leq q, a \wedge q=1} F(a / q)=\sum_{d \mid q} \mu(q / d) \sum_{1 \leq m \leq d} F(m / d) .
$$

Let $1 \leq q<2^{s}$. Recall that (see e.g. Tenenbaum [16] p. 83) there exists $c>0$ such that

$$
\sum_{d \mid q}|\mu(d)|=2^{\omega(q)} \leq 2^{c \frac{\log q}{\log \log q}}=O\left(q^{\varepsilon}\right)
$$

where $\omega(q)$ is the number of prime divisors of $q$.

We shall apply (6.5) with $F\left(\frac{m}{d}\right)=\int_{-1 / 2}^{1 / 2} \widehat{w}_{n}(x) \eta(Q x) \widehat{g}(m / d+x) \mathrm{e}^{2 i \pi j(m / d+x)} d x$. We combine it with Lemma 6.2 with $Q=4^{s} M$ if $p \neq 2$ and Lemma 6.3 if $p=2$.

We shall now deal with families of sequences $\left(w_{n}\right)_{n \geq 1}$ rather than with a single sequence. In particular, $\left(\left(w_{n, q}\right)_{n \geq 1}\right)_{q \geq 1}$ will be a family of elements of $\ell^{1}(\mathbb{Z})$, such that for every $p>1$, there exists $C_{p}>0$ such that for every integer $s \geq 1$, there exists $K_{s}$ such that,

$$
\left\|\sup _{n \geq 1}\left|w_{n, q} * g\right|\right\|_{\ell^{p}(\mathbb{Z})} \leq C_{p} K_{s}\|g\|_{\ell^{p}(\mathbb{Z})} \quad \forall g \in \ell^{p}(\mathbb{Z}), \forall 2^{s-1} \leq q<2^{s} .
$$

Corollary 6.5. There exists $C>0$ such that for every $s \geq 1$, every $g \in \ell^{2}(\mathbb{Z})$ and every family $\left(\left(w_{n, q}\right)_{n \geq 1}\right)_{2^{s-1} \leq q<2^{s}}$ of elements of $\ell^{1}(\mathbb{Z})$ satisfying (6.6), we have

$$
\begin{array}{r}
\sum_{2^{s-1} \leq q<2^{s}}\left\|\sup _{n} \mid \sum_{1 \leq a \leq q, a \wedge q=1} \int_{-1 / 2}^{1 / 2} \widehat{w}_{n, q}(x) \eta_{s}(x) \widehat{g}(a / q+x) \mathrm{e}^{2 i \pi j(a / q+x)} d x\right\|_{\ell^{2}(\mathbb{Z}, d j)} \\
\leq C K_{s} 2^{(\varepsilon+1 / 2) s}\|g\|_{\ell^{2}} .
\end{array}
$$

Proof. For every $s \geq 1, \eta_{s-1} \equiv 1$ on $\left[-1 /\left(M 4^{s}\right), 1 /\left(M 4^{s}\right)\right]$, hence $\eta_{s-1} \eta_{s}=\eta_{s}$. Moreover the functions $\left\{x \rightarrow \eta_{s-1}(x-a / q)\right\}_{2^{s-1} \leq q<2^{s}, 1 \leq a \leq q, a \wedge q=1}$ have disjoint supports.

Indeed, let $s \geq 1,2^{s-1} \leq q<2^{s}$ and $1 \leq a \leq q$. Let $x \in[0,1]$ be such that $\eta_{s}(x-a / q)>0$. Then, $|x-a / q| \leq \frac{1}{2 \cdot 4^{s} M}$ and if $2^{s-1} \leq q^{\prime}<2^{s}$ and $1 \leq a^{\prime} \leq q^{\prime}$, we have

$$
\left|x-a^{\prime} / q^{\prime}\right| \geq\left|a / q-a^{\prime} / q^{\prime}\right|-|x-a / q| \geq \frac{1}{2 \cdot 4^{s}}-\frac{1}{4^{s} M} \geq \frac{1}{2 \cdot 4^{s} M} .
$$


Hence $\eta_{s}\left(x-a^{\prime} / q^{\prime}\right)=0$. In particular, writing

$$
\widehat{g}_{q}(x)=\sum_{1 \leq a \leq q} \eta_{s-1}\left(x-\frac{a}{q}\right) \widehat{g}(x),
$$

we see that

$$
\sum_{1 \leq a \leq q, a \wedge q=1} w_{n, q}\left(x-\frac{a}{q}\right) \eta_{s}\left(x-\frac{a}{q}\right) \widehat{g}(x) \mathrm{e}^{2 i \pi j x}=\sum_{1 \leq a \leq q, a \wedge q=1} w_{n, q}\left(x-\frac{a}{q}\right) \eta_{s}\left(x-\frac{a}{q}\right) \widehat{g}_{q}(x) \mathrm{e}^{2 i \pi j x} .
$$

Applying Corollary 6.4 and using that $\left\|g_{q}\right\|_{\ell^{2}}=\left\|\widehat{g}_{q}\right\|_{2}$, we infer that

$$
\begin{aligned}
& \left.\sum_{2^{s-1} \leq q<2^{s}}|| \sup _{n}\right|_{1 \leq a \leq q, a \wedge q=1} \int_{-1 / 2}^{1 / 2} w_{n, q}(x) \eta_{s}(x) \widehat{g}(a / q+x) \mathrm{e}^{2 i \pi j(a / q+x)} d x \mid \|_{\ell^{2}(\mathbb{Z}, d j)} \\
\leq & C K_{s} 2^{\varepsilon s} \sum_{2^{s-1} \leq q<2^{s}}\left\|\widehat{g}_{q}\right\|_{2} \leq C K_{s} 2^{(\varepsilon+1 / 2) s}\left(\sum_{2^{s-1} \leq q<2^{s}}\left\|\widehat{g}_{q}\right\|_{2}^{2}\right)^{1 / 2} \\
= & C K_{s} 2^{(\varepsilon+1 / 2) s}\left(\sum_{2^{s-1} \leq q<2^{s}} \sum_{1 \leq a \leq q, a \wedge q=1} \int_{0}^{1}|\widehat{g}(x)|^{2} \eta_{s-1}^{2}(x-a / q) d x\right)^{1 / 2} \\
\leq & C K_{s} 2^{(\varepsilon+1 / 2) s}\|g\|_{\ell^{2}}
\end{aligned}
$$

where we used the above mentionned disjointness.

Corollary 6.6. Let $p>1$. For every $\delta>1 / p$, there exists $C_{p, \delta}>0$ such that for every $s \geq 1$ and every $g \in \ell^{p}(\mathbb{Z})$

$\left\|\sum_{2^{s-1} \leq q<2^{s}} \sup _{n} \mid \sum_{1 \leq p \leq q, a \wedge q=1} \int_{-1 / 2}^{1 / 2} \widehat{w}_{n, q}(x) \eta_{s}(x) \widehat{g}(a / q+x) \mathrm{e}^{2 i \pi j(a / q+x)} d x\right\|_{\ell^{p}(\mathbb{Z}, d j)} \leq C_{p, \delta} K_{s} 2^{s \delta}\|g\|_{\ell^{p}}$.

Remark 6.7. Notice that the sum is inside the norm that time.

Proof. Let $s \geq 1$. Consider the following sub-additive and bounded (by Corollaries 6.4 and 6.5) operators on $\ell^{r}(\mathbb{Z}), 1<r \leq 2$ :

$$
\mathbb{L}_{s}(g)=\sum_{2^{s-1} \leq q<2^{s}} \sup _{n}\left|\sum_{1 \leq a \leq q, a \wedge q=1} \int_{-1 / 2}^{1 / 2} \widehat{w}_{n, q}(x) \eta_{s}(x) \widehat{g}(a / q+x) \mathrm{e}^{2 i \pi j(a / q+x)} d x\right| .
$$

Let $1<p<2$ and chose any $r \in(1, p)$. Let $\lambda \in(0,1)$ be the unique real number such that $1 / p=\lambda / r+(1-\lambda) / 2$.

By the Marcinkiewicz interpolation theorem (see e.g. Zygmund [21, Th 4.6, Ch. XII, Vol. II]), there exists $C_{p, r}>0$ such that

$$
\left\|\mathbb{L}_{s} g\right\|_{\ell^{p}} \leq C_{p, r} K_{s} 2^{s(2+\varepsilon-1 / r) \lambda} 2^{(1-\lambda)(\varepsilon+1 / 2) s}\|g\|_{\ell^{p}}=C_{p, r} 2^{s[1+\varepsilon-1 / r) \lambda+(1-\lambda) \varepsilon]} 2^{(1+\lambda) s / 2}\|g\|_{\ell^{p}} .
$$

Taking $r$ close enough to 1 , we may assume that $1+\varepsilon-1 / r) \lambda+(1-\lambda) \varepsilon \leq 3 \varepsilon$.

Notice that $\lambda=\frac{r(2-p)}{p(2-r)}$ and that $(1+\lambda) / 2=\frac{r+p-r p}{p(2-r)} \underset{r \rightarrow 1}{\longrightarrow} 1 / p$. The result follows since $\varepsilon$ may be taken arbitrary small.

\section{Approximation result}

In this section, we explain how to derive from the estimates on exponential sums, good approximation results with suitable Fourier kernels to which we can apply the previous maximal inequalities. 
7.1. We use the notation (6.1), (6.4). Let $0<\tau \leq 1$ be a parameter to be chosen later. Assume that we have a collection $\left(\psi_{n, q}\right)_{n \geq 1, q \geq 0}$ of complex-valued 1-periodic functions on $\mathbb{R}$ such that there exists $C>0$ such that for every $x \in[-1 / 2,1 / 2]$

$$
\left|\psi_{n, q}(x)\right| \leq \frac{C}{(q+1)^{\tau}} \min \left(1, \frac{1}{n|x|}\right) .
$$

Let $\left(P_{n}\right)_{n \geq 1}$ and $\left(Q_{n}\right)_{n \geq 1}$ be two non-decreasing sequences of integers. Assume that there exist $R, S>1$ such that for every $n \geq 2$,

$$
P_{n} \geq R(\log n)^{S / \tau} \quad \text { and } \quad 16 M P_{n}^{2} \leq Q_{n} \leq \frac{n}{(\log n)^{S(1+1 / \tau)}} .
$$

In particular,

$$
Q_{n} \geq 16 M P_{n} \geq 16 M R(\log n)^{S / \tau} \geq(\log n)^{S / \tau} .
$$

Denote

$$
\mathcal{M}\left(P_{n}, Q_{n}\right)=\mathcal{M}_{n}:=\left\{x \in[0,1]: \exists 0 \leq a \leq q \leq P_{n}:|x-a / q| \leq 1 / Q_{n}\right\} .
$$

Notice that, because of (7.2), if $x \in \mathcal{M}_{n}, x \neq 0$, there exist unique numbers $a_{n}(x)$ and $q_{n}(x)$ with $1 \leq a_{n} \leq q_{n}$ and $a_{n} \wedge q_{n}=1$ and such that $\left|x-a_{n}(x) / q_{n}(x)\right| \leq 1 / Q_{n}$. Let us also define $a_{n}(0)=0$ and $q_{n}(0)=1$.

Finally, define functions $\varphi_{n}$ on $[0,1]$ by

$$
\varphi_{n}(x):=\psi_{n, 0}(x) \eta_{0}(x)+\sum_{s=1}^{\infty} \sum_{2^{s-1} \leq q<2^{s}} \sum_{1 \leq a \leq q, a \wedge q=1} \psi_{n, q}(x-a / q) \eta_{s}(x-a / q) .
$$

Notice that for any fixed $s$, the functions

$$
x \mapsto \eta_{s}(x-a / q), \quad 2^{s-1} \leq q<2^{s}, 1 \leq a \leq q, a \wedge q=1,
$$

have disjoint supports. Hence, by (7.1) the series defining $\left(\varphi_{n}\right)_{n \geq 1}$ are uniformly convergent.

We shall need the following technical lemma, which is essentially due to Bourgain.

Lemma 7.1. Let $\left(T_{n}\right)_{n \geq 1}$ be complex-valued functions on $[0,1]$, such that there exists $C>0$ and $\gamma>0$ such that for every $n \geq 2$,

$$
\begin{array}{r}
\left|T_{n}(x)-\psi_{n, q_{n}}\left(x-a_{n} / q_{n}\right)\right| \leq \frac{C}{(\log n)^{\gamma}} \quad \forall x \in \mathcal{M}_{n} \\
\left|T_{n}(x)\right| \leq \frac{C}{(\log n)^{\gamma}} \quad \forall x \in[0,1] \backslash \mathcal{M}_{n} .
\end{array}
$$

Then, there exists $\widetilde{C}>0$ such that for every $n \geq 2$ and every $x \in[0,1]$,

$$
\left|T_{n}(x)-\varphi_{n}(x)\right| \leq \frac{\widetilde{C}}{(\log n)^{\min (\gamma, S)}} .
$$

Proof. 1. We start with the case where $x \in[0,1] \backslash \mathcal{M}_{n}$. By (7.6), it suffices to estimate $\left|\varphi_{n}\right|$. By assumption, $\min (x, 1-x) \geq \frac{1}{Q_{n}}$. Hence,

$$
\left|\psi_{n, 0}(x)\right| \leq \frac{C Q_{n}}{n} \leq \frac{C}{(\log n)^{S(1+1 / \tau)}} .
$$

By Dirichlet's principle, there exists $1 \leq a \leq q \leq Q_{n}$, with $a \wedge q=1$ such that

$$
\left|x-\frac{a}{q}\right| \leq \frac{1}{q Q_{n}} .
$$


Let $1 \leq a^{\prime} \leq q^{\prime}$ with $a^{\prime} \wedge q^{\prime}=1$ and $a^{\prime} / q^{\prime} \neq a / q$. Then, if $q^{\prime} \leq \frac{(\log n)^{S / \tau}}{2}$, using (7.2) and (7.3), we have

$$
\left|x-\frac{a^{\prime}}{q^{\prime}}\right| \geq \frac{1}{q q^{\prime}}-\frac{1}{q Q_{n}}=\frac{1}{q}\left(\frac{1}{q^{\prime}}-\frac{1}{Q_{n}}\right) \geq \frac{1}{q(\log n)^{S / \tau}} \geq \frac{1}{Q_{n}(\log n)^{S / \tau}} \geq \frac{(\log n)^{S}}{n} .
$$

Hence, when $q^{\prime} \leq \frac{(\log n)^{S / \tau}}{2}$,

$$
\left|\psi_{n, q^{\prime}}\left(x-\frac{a^{\prime}}{q^{\prime}}\right)\right| \leq \frac{C}{\left(q^{\prime}+1\right)^{\tau}(\log n)^{S}}
$$

So, using (7.4), we obtain

$$
\left|\varphi_{n}(x)\right| \leq \frac{1}{(\log n)^{S(1+1 / \tau)}}+\left|\psi_{n, q}(x)\right|+\frac{C}{(\log n)^{S}} \sum_{s: 2^{s} \leq(\log n)^{S / \tau}} 2^{-s \tau}+C \sum_{s: 2^{s} \geq(\log n)^{S / \tau}} 2^{-s \tau} .
$$

Now, since $x \in[0,1] \backslash \mathcal{M}_{n}, q \geq P_{n}$ and $\left|\psi_{n, q}(x)\right| \leq C /(q+1)^{\tau}=O\left((\log n)^{S}\right)$. Hence the lemma is proved in that case.

2. Assume now that $x \in \mathcal{M}_{n}$. Suppose $x \neq 0$. By assumption, $\left|x-a_{n}(x) / q_{n}(x)\right| \leq 1 / Q_{n}$ and $q_{n}(x) \leq P_{n}$. Hence, if $s \geq 1$, is such that $2^{s-1} \leq q_{n}(x)<2^{s}$, we have

$$
\left|x-a_{n}(x) / q_{n}(x)\right| \leq 1 / Q_{n} \leq \frac{8 M P_{n}^{2}}{Q_{n}} \cdot \frac{1}{8 M P_{n}^{2}} \leq \frac{1}{2 M 4^{s}} .
$$

In particular, $\eta_{s}\left(x-a_{n}(x) / q_{n}(x)\right)=1$. If $x=0, \eta_{0}(0)=1$.

Let $1 \leq a^{\prime} \leq q^{\prime}$ with $a^{\prime} \wedge q^{\prime}=1$ and $a^{\prime} / q^{\prime} \neq a / q$. Then, if $q^{\prime} \leq P_{n}$, using (7.2)

$$
\left|x-\frac{a^{\prime}}{q^{\prime}}\right| \geq \frac{1}{q_{n}(x) q^{\prime}}-\frac{1}{Q_{n}} \geq \frac{1}{P_{n}^{2}}-\frac{1}{Q_{n}} \geq \frac{8 M-1}{Q_{n}},
$$

and $\left|\psi_{n, q^{\prime}}\left(x-\frac{a^{\prime}}{q^{\prime}}\right)\right| \leq \frac{8 M-1}{\left(q^{\prime}+1\right)^{\tau}(\log n)^{S(1+1 / \tau)}}$, by $(7.2)$.

Finally, we obtain

$$
\left|\varphi_{n}(x)-T_{n}(x)\right| \leq \frac{C}{(\log n)^{\gamma}}+\frac{8 M-1}{(\log n)^{1+1 / \tau}} \sum_{s: 2^{s} \leq P_{n}} 2^{-\tau s}+C \sum_{s: 2^{s}>P_{n}} 2^{-\tau s},
$$

which proves the lemma in that case.

7.2. Let us assume from now that there exists a sequence $\left(w_{n, q}\right)_{n \geq 1, q \geq 0}$ of elements of $\ell^{1}$ such that assumption (7.1) is satisfied with the choice

$$
\psi_{n, q}=\widehat{w}_{n, q} \quad n \geq 1, q \geq 0 .
$$

Introduce the following assumption.

For every $p>1$, there exists $C_{p}>0$ such that

$$
\left\|\sup _{n \geq 1}\left|w_{n, q} * g\right|\right\|_{\ell^{p}} \leq \frac{C_{p}}{q^{\tau}}\|g\|_{\ell^{p}} \quad \forall g \in \ell^{p} .
$$

Proposition 7.2. Let $\left(K_{n}\right)_{n \geq 1} \subset \ell^{1}$, with $\sup _{n \geq 1}\left\|K_{n}\right\|_{\ell^{1}}<\infty$. Assume that $T_{n}:=\widehat{K}_{n}$ satisfies (7.5) and (7.6), for some $\gamma>1 / 2$. Assume moreover that (7.9) holds. Then, for every $p \in$ $\left(\frac{1}{\tau}+\frac{2-1 / \tau}{2 \min (\gamma, S)}, 2\right]$, there exists $C_{p}>0$, such that

$$
\left\|\sup _{n \geq 1}\left|K_{2^{n}} * g\right|\right\|_{\ell^{p}} \leq C_{p}\|g\|_{\ell^{p}} \quad \forall g \in \ell^{p} .
$$

Remark 7.3. According to Section 4, Proposition 7.2 provides the maximal inequality for the kernel $K_{n}$, and thereby in any measurable dynamical system.

For the proof, we will need the following Lemma. Let $L_{n}$ be the inverse Fourier transform of $\varphi_{n}$, which is made possible because of the introduction of the smooth function $\eta$. 
Lemma 7.4. For every $p>1 / \tau$, there exists $C_{p}>0$ such that for every $g \in \ell^{p}$,

$$
\left\|\sup _{n \geq 1}\left|g * L_{n}\right|\right\|_{\ell^{p}} \leq C_{p}\|g\|_{\ell^{p}} .
$$

Proof of Lemma 7.4. Let $r>1 / \tau$. We apply Corollary 6.6 (as $\left.\psi_{n, q}=\widehat{w}_{n, q}\right)$ to obtain that for every $\delta>1 / r$ and every $g \in \ell^{r}$,

$$
\begin{array}{r}
\left\|\sum_{2^{s-1} \leq q<2^{s}} \sup _{n}\left|\sum_{1 \leq p \leq q, a \wedge q=1} \int_{-1 / 2}^{1 / 2} \widehat{w}_{n, q}(x) \eta_{s}(x) \widehat{g}(a / q+x) \mathrm{e}^{2 i \pi j(a / q+x)} d x\right|\right\|_{\ell^{r}(\mathbb{Z}, d j)} \\
\leq C_{r, \delta} 2^{s(\delta-\tau)}\|g\|_{\ell^{r}} .
\end{array}
$$

We may chose $\delta<\tau$, so that $\sum_{s \geq 0} 2^{s(\delta-\tau)}<\infty$. Summing the estimates (7.11) over $s \geq 1$ we infer that for every $g \in \ell^{r}$,

$$
\left\|\sup _{n \geq 1} \int_{-1 / 2}^{1 / 2} \widehat{L}_{n}(x) \widehat{g}(x) \mathrm{e}^{2 i \pi j x} d x\right\|_{\ell^{r}(\mathbb{Z})} \leq C_{r}\|g\|_{\ell^{r}(\mathbb{Z})} .
$$

Taking inverse Fourier transform we see that Lemma 7.4 is true.

Proof of Proposition 7.2. By Lemma 7.1, since (7.5) and (7.6) are satisfied, we see that (7.7) holds. Hence, we have

$$
\left\|\widehat{K}_{n}-\widehat{L}_{n}\right\|_{\infty} \leq \frac{C}{(\log n)^{\min (\gamma, S)}} \quad \forall n \geq 2 .
$$

Then, we infer that for every $f \in \ell^{2}(\mathbb{Z})$,

$$
\left\|f *\left(K_{n}-L_{n}\right)\right\|_{\ell^{2}(\mathbb{Z})} \leq \frac{C}{(\log n)^{\min (\gamma, S)}}\|f\|_{\ell^{2}(\mathbb{Z})} \quad \forall n \geq 2 .
$$

Let $2>p>1 / \tau$, be fixed for the moment. Since $\sup _{n>1}\left\|K_{n}\right\|_{\ell^{1}(\mathbb{Z})} \leq C$, we see that for every $n \geq 1$ and for every $r \geq 1$ and $g \in \ell^{r}(\mathbb{Z})$ (using Young's inequalities), $\left\|K_{n} * g\right\|_{\ell^{r}(\mathbb{Z})} \leq C\|g\|_{\ell^{r}(\mathbb{Z})}$. Hence, by (7.13) and Lemma 7.4, we see that, for every $n \geq 0$ and every $r>1 / \tau$,

$$
\begin{aligned}
& \left\|K_{2^{n}} * g-L_{2^{n}} * g\right\|_{\ell^{2}(\mathbb{Z})} \leq \frac{C}{n^{\min (\gamma, S)}}\|g\|_{\ell^{2}(\mathbb{Z})} \quad \forall g \in \ell^{2}(\mathbb{Z}), \\
& \left\|K_{2^{n}} * g-L_{2^{n}} * g\right\|_{\ell^{r}(\mathbb{Z})} \leq C_{r}\|g\|_{\ell^{r}(\mathbb{Z})} \quad \forall g \in \ell^{2}(\mathbb{Z})
\end{aligned}
$$

Let $1 / \tau<r<p$. Interpolating, we deduce that there exists $C_{p, r}$ such that for every $n \geq 0$,

$$
\left\|K_{2^{n}} * g-L_{2^{n}} * g\right\|_{\ell^{p}(\mathbb{Z})} \leq \frac{C_{r, p}}{n^{\sigma}}\|g\|_{\ell^{r}(\mathbb{Z})} \quad \forall g \in \ell^{p}(\mathbb{Z}),
$$

with $\sigma=\frac{2 \tilde{\gamma}(p-r)}{p(2-r)}$ and $\tilde{\gamma}=\min (\gamma, S)$.

Notice that

$$
\sigma-\frac{1}{p}=\frac{1}{p}\left(\frac{2 \tilde{\gamma}(p-r)}{2-r}-1\right) \underset{r \rightarrow 1 / \tau}{\longrightarrow} \frac{2 \tilde{\gamma}(p-1 / \tau)+1 / \tau-2}{p(2-1 / \tau)} .
$$

Since $p>\frac{1}{\tau}+\frac{2-1 / \tau}{2 \min (\gamma, S)}$, we may chose $r$ close enough to $1 / \tau$, such that $\sigma>1 / p$. In particular for that choice,

$$
\left\|\sup _{n \geq 0}\left|K_{2^{n}} * g-L_{2^{n}} * g\right|\right\|_{\ell^{p}(\mathbb{Z})} \leq\left(\sum_{n \geq 0}\left\|K_{2^{n}} * g-L_{2^{n}} * g\right\|_{\ell^{p}(\mathbb{Z})}^{p}\right)^{1 / p} \leq C_{p, r}\|g\|_{\ell^{p}(\mathbb{Z})} \quad \forall g \in \ell^{p}(\mathbb{Z}) .
$$


7.3. We now establish an estimate of the type (4.3) in order to prove the convergence almost everywhere. Recall that for $\rho>1$, we have noted $I_{\rho}=\left\{\left[\rho^{n}\right]: n \in \mathbb{N}\right\}$. Introduce the following assumption:

For every $\rho>1$ and every sequence $\left(N_{j}\right)_{j \geq 1}$, with $N_{j+1} \geq 2 N_{j}$, there exists $C>0$ such that,

$$
\sum_{j \geq 1}\left\|\sup _{\substack{N_{j} \leq N \leq N_{j+1} \\ N \in I_{\rho}}}\left|\left(w_{N, q}-w_{N_{j}, q}\right) * g\right|\right\|_{\ell^{2}(\mathbb{Z})}^{2} \leq \frac{C}{q^{\tau}}\|g\|_{2} \quad \forall g \in \ell^{2} .
$$

Theorem 7.5. Let $\left(K_{n}\right)_{n \geq 1} \subset \ell^{1}$. Assume that $T_{n}:=\widehat{K}_{n}$ satisfies (7.5) and (7.6), for some $\gamma>1 / 2$. Assume moreover that (7.14) holds. Then, for every $\rho>1$ and every sequence $\left(N_{j}\right)_{j \geq 1}$, with $N_{j+1} \geq 2 N_{j}$,

$$
\sum_{j=1}^{J}\left\|\sup _{N_{j} \leq N \leq N_{j+1}, N \in I_{\rho}}\left|\left(K_{N}-K_{N_{j}}\right) * g\right|\right\|_{\ell^{2}(\mathbb{Z})}^{2}=o(J) .
$$

Remark 7.6. According to Section 4 the convergence almost everywhere now follows from Theorem 7.5 .

Proof. The proof follows closely the argument p. 220 in Bourgain [3]. Let $\rho>1$. Let $\left(N_{j}\right)_{j \in \mathbb{N}} \subset I_{\rho}$ be an increasing sequence with $N_{j+1}>2 N_{j}$. For every $j \in \mathbb{N}$, define a maximal operator by

$$
M_{j} f=M_{j, \rho} f:=\sup _{N_{j} \leq N<N_{j+1}, N \in I_{\rho}}\left|f * K_{N}-f * K_{N_{j}}\right| \quad \forall f \in \ell^{2}(\mathbb{Z}) .
$$

As in the previous proof we define $L_{n}$ as the inverse Fourier transform of $\varphi_{n}$. Notice that, for every $f \in \ell^{2}(\mathbb{Z})$,

$$
\begin{aligned}
M_{j} & \leq \sup _{N_{j} \leq N<N_{j+1}, N \in I_{\rho}}\left|f * L_{N}-f * L_{N_{j}}\right|+2 \sup _{N_{j} \leq N<N_{j+1}, N \in I_{\rho}}\left|f *\left(L_{N}-K_{N}\right)\right| \\
& :=\widetilde{M}_{j}+2 \sup _{N_{j} \leq N<N_{j+1}, N \in I_{\rho}}\left|f *\left(L_{N}-K_{N}\right)\right| .
\end{aligned}
$$

Hence,

$$
\sum_{1 \leq j \leq J}\left\|M_{j} f\right\|_{\ell^{2}}^{2} \leq 4\left(\sum_{1 \leq j \leq J}\left\|\widetilde{M}_{j} f\right\|_{\ell^{2}}^{2}+\sum_{N \in I_{\rho}}\left|f *\left(L_{N}-K_{N}\right)\right|^{2}\right) .
$$

Using (17.12), we see that $\left\|\widehat{L}_{\left[\rho^{n}\right]}-\widehat{K}_{\left[\rho^{n}\right]}\right\|_{\infty} \leq \frac{C}{n^{\gamma} \log \rho}$, with $\tilde{\gamma}=\min (\gamma, S)>1 / 2$. Hence

$$
\sum_{N \in I_{\rho}}\left\|f *\left(L_{N}-K_{N}\right)\right\|_{\ell^{2}(\mathbb{Z})}^{2} \leq\|f\|_{\ell^{2}(\mathbb{Z})}^{2} \sum_{N \in I_{\rho}}\left\|\widehat{L}_{N}-\widehat{K}_{N}\right\|_{\infty}^{2}<\infty ;
$$

Hence, it is enough to prove the theorem with $\left(\widetilde{M}_{j}\right)$ in place of $\left(M_{j}\right)$. Let $t=t(J)$ be an integer to be chosen later. Define $R_{N}$ through its Fourier transform, i.e.

$$
\widehat{R}_{N}(x):=\widehat{w}_{n, 0}(x) \eta_{0}(x)+\sum_{1 \leq s \leq t} \sum_{2^{s-1} \leq q<2^{s}} \sum_{1 \leq a \leq q, a \wedge q=1} \widehat{w}_{n, q}(x-a / q) \eta_{s}(x-a / q) .
$$

It follows from (7.11) that for every $1 / 2<\delta<\tau$,

$$
\left\|\sup _{N \in I_{\rho}} \mid f *\left(L_{N}-R_{N}\right)\right\|_{\ell^{2}(\mathbb{Z})} \leq C 2^{(\delta-\tau) t} .
$$

In particular,

$$
\sum_{1 \leq j \leq J}\left\|\widetilde{M}_{j} f\right\|_{\ell^{2}}^{2} \leq \sum_{1 \leq j \leq J}\left\|\sup _{N_{j} \leq N<N_{j+1}, N \in I_{\rho}}\left|f * R_{N}-f * R_{N_{j}}\right|\right\|_{\ell^{2}}^{2}+C J 2^{2(\delta-\tau) t}
$$

Define $g_{s, \frac{a}{q}}$ by $\widehat{g}_{s, \frac{a}{q}}(x)=\eta_{s}(x) f\left(x+\frac{a}{q}\right)$ and $g_{0}(x)=\eta_{0}(x) f(x)$. Then, using the change of variable $x \rightarrow x+\frac{a}{q}$, for every $k \in \mathbb{Z}$, we have

$$
f * R_{N}(k)=\int_{-1 / 2}^{1 / 2} \widehat{f}(x) \widehat{R}_{N}(x) \mathrm{e}^{-2 i \pi k x} d x=\int_{-1 / 2}^{1 / 2} \widehat{g}_{0}(x) \widehat{w}_{N, 0}(x) \mathrm{e}^{-2 i \pi k x} d x
$$




$$
+\sum_{1 \leq s \leq t} \sum_{2^{s-1} \leq q<2^{s}} \frac{1}{q} \sum_{1 \leq a \leq q, a \wedge q=1} \mathrm{e}^{-2 i k \pi \frac{a}{q}} \int_{-1 / 2}^{1 / 2} \widehat{g}_{s, \frac{a}{q}}(x) \widehat{w}_{N, q}(x) \mathrm{e}^{-2 i \pi k x} d x .
$$

Hence,

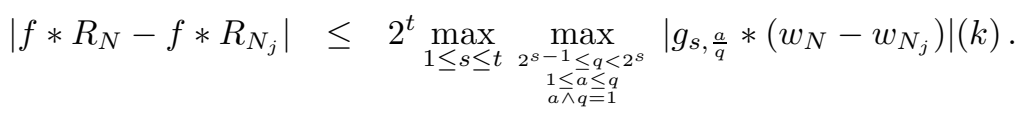

Combining (7.16), (7.17), (7.18) and (7.14), we infer that

$$
\sum_{1 \leq j \leq J}\left\|\widetilde{M}_{j} f\right\|_{\ell^{2}}^{2} \leq C 2^{t}\|f\|_{\ell^{2}(\mathbb{Z})}+C J 2^{2(\delta-\tau) t},
$$

which is $o(J)$ if we chose for instance $t(J)=[\log \log J]$, and the theorem is proved.

\section{Proof of Theorem 1.1}

Firstly, we prove the dominated ergodic theorem for the weights $\left(d_{n}\right)_{n \geq 1}$. In this case, since $D_{n}$ does not grow too fast, it suffices to deal with positive functions and to prove a maximal inequality along the dyadic integers.

For every $n \geq 2$ and every $q \geq 1$, define

$$
w_{n, q}:=\frac{1}{q n \log n} \sum_{1 \leq k \leq n} \log k \delta_{k}+\frac{2(\gamma-1-\log q)}{n \log n} \sum_{1 \leq k \leq n} \delta_{k},
$$

and

$$
\psi_{n, q}(x):=\hat{w}_{n, q}(x)=\frac{1}{q n} \sum_{1 \leq k \leq n} \log k \mathrm{e}^{i k x}+\frac{2(\gamma-1-\log q)}{n \log n} \sum_{1 \leq k \leq n} \mathrm{e}^{i k x} .
$$

Using the well-known estimate $\frac{1}{n}\left|\sum_{1 \leq k \leq n} \mathrm{e}^{i k x}\right| \leq \min \left(1, \frac{1}{|n x|}\right)$ and Abel summation to deal with the first term in (8.1), we see that (7.1) holds for any $\tau \in[0,1)$.

It is also well-known that (Reference ??), writing $\kappa_{n}:=\frac{1}{n} \sum_{1 \leq k \leq n} \delta_{k}$, for every $p>1$, there exists $C_{p}>0$ such that

$$
\left\|\sup _{n \geq 1} \mid \kappa_{n} * g\right\|_{\ell^{p}} \leq C_{p}\|g\|_{\ell^{p}} \quad \forall g \in \ell^{r} .
$$

Since $\frac{1}{n \log n} \sum_{1 \leq k \leq n} \log k \delta_{k} \leq \kappa_{n}$, we infer that (7.9) holds far any $\tau \in[0,1)$.

Let $S>1$. For every $n \geq 2$ define

$$
P_{n}:=\left[( \operatorname { l o g } n ) ^ { 3 S } \left[, \quad Q_{n}=\left[n /(\log n)^{2 S}\right] .\right.\right.
$$

Then, by (5.4) of Lemma 5.2 and by Lemma 5.4 we see that (7.5) and (17.6) holds for $T_{n}(x):=$ $D_{n}(x) / D_{n}$, with $\gamma=S$.

Hence, by Proposition 7.2 and Calderon's transference principle, we see that $\left(d_{n}\right)_{n \geq 1}$ is a good weight for the dominated ergodic theorem in $L^{p}$ for every $p \in\left[1 / \tau+\frac{2-1 / \tau}{2 S}, 2\right]$. Since we may take $\tau$ arbitrary close to 1 and $S$ arbitrary large, the dominated ergodic theorem holds for every $p>1$ as well.

Secondly, we shall prove an oscillation inequality in $L^{2}$. The proof is exactly as above except that we take $w_{n, q}:=\frac{1}{q n} \sum_{1 \leq k \leq n} \delta_{k}$, that we make use (5.5) of Lemma 5.2 and that we apply Theorem 7.5 (instead of Proposition 7.2). 


\section{Open Problems.}

We conclude by listing some natural problems arising from this work.

Problem 9.1 (Extension to $L^{1, \infty}$ ). Does our main Theorem 1.1 remain true in $L^{1}$ ? Same question with Theorem 1.2 with the $\theta$ function in place of the divisor function.

Problem 9.2 (Square function). Let $\mathcal{N}=\left\{n_{j}, j \geq 1\right\}$ be an increasing sequence of positive integers, and define for any $f \in L^{2}$,

$$
S_{\mathcal{N}}(f)=\left(\sum_{p=1}^{\infty}\left\|A_{n_{j+1}}^{\tau}(f)-A_{n_{j}}^{\tau}(f)\right\|_{2}^{2}\right)^{1 / 2},
$$

recalling that $A_{n} f:=\frac{1}{W_{n}} \sum_{k=1}^{n} w_{k} f \circ \tau^{k}$. When $w_{k}=d(k)$, is it true that

$$
\left\|S_{\mathcal{N}}(f)\right\|_{2} \leq C(\mathcal{N})\|f\|_{2}
$$

for any $f \in L^{2}$ ? Is this further true for any increasing sequence of positive integers $\mathcal{N}$ ?

Problem 9.3 (Spectral Regularization). Can one associate to $V_{n}(\theta)=\frac{1}{D_{n}} \sum_{k=1}^{n} d(k) \mathrm{e}^{2 i \pi n \theta}$, a regularizing kernel $Q(\theta, y)$ on $[0,1)^{2}$ so that for any $f \in L^{2}$, with spectral measure $\mu_{f}$ (relatively to the operator $T f=f \circ \tau)$, the new measure defined by

$$
\widehat{\mu}_{f}(d y)=\left(\int_{0}^{1} Q(\theta, y) \mu_{f}(d \theta)\right) d y
$$

verifies

$$
\left.\left.\left\|A_{n}^{T} f-A_{m}^{T} f\right\|^{2} \leq \widehat{\mu}_{f}\{\rceil \frac{1}{m}, \frac{1}{n}\right\rceil\right\}
$$

for any integers $m \geq n \geq 1$ ? Such an inequality immediately provides a control on the square function associated to these averages. So is the case for usual ergodic averages where the corresponding oscillations functions can be controlled similarly. We refer to [17] Part I, Section 1.4 concerning this notion and the related results.

Problem 9.4 (Extensions to other arithmetical functions). Can one establish the validity of Theorem 1.1 for other arithmetical functions? Examples can be function $r(n)$ counting the number of ways to write $n$ as a sum of two squares, the Piltz divisor function $d_{k}(n)$ counting the number of ways to write $n$ as a product of $k$ factors (in the latter case we do not believe that it is an easy task). In each of these cases, the validity (in $L^{1}$ ) of the strong law of large numbers was recently established in [2]. One may also consider the same question for the multiplicative function $R(u)=\#\left\{(\delta, d) \in \mathbb{N}^{2}:[d, \delta]=u\right\}$

\section{References}

[1] P. T. Bateman, S. Chowla, Some special trigonometrical series related to the distribution of primes, J. London Math. Soc., 38, 372-374, (1963).

[2] I. Berkes, W. Müller, M. Weber, On the strong law of large numbers and arithmetic functions, Indagationes Math. 23, 547-555, (2012).

[3] J. Bourgain, On the maximal ergodic theorem for certain subsets of the integers, Israel J. Math. 61, 39-72, (1988).

[4] J. Bourgain, Pointwise ergodic theorems for arithmetic sets, with an appendix on return time sequences, jointly with H. Furstenberg, Y. Katznelson, D. Ornstein, Inst. Hautes Études Sci. Publ. Math. 69, 5-45, (1988).

[5] J. Bourgain, An approach to pointwise ergodic theorems, Geometric aspects of functional analysis (1986/87), 204-223, Lecture Notes in Math., 1317, Springer, Berlin, (1988).

[6] H. Davenport, On some infnite series involving arithmetical functions, Quart. J. Math., Oxf. Ser. 8, 8-13, (1937).

[7] H. Davenport, On some infnite series involving arithmetical functions II, Quart. J. Math., Oxf. Ser. 8, 313-320, (1937).

[8] H. Delange, Sur des formules de Atle Selberg, Acta Arith. 19, 105-146, (1971).

[9] C. Demeter and A. Quas, Weak-L $L^{1}$-estimates and ergodic theorems, New York J. Math., 10, 169-174, (2004).

[10] G. Hardy, J. E. Littlewood (1930) A maximal theorem with functions-theoretic applications, Acta Math. 54, 81-116.

[11] R. Jones, R. Kaufman, J. Rosenblatt and M. Wierdl, Oscillation in ergodic theory, Ergodic Theory Dynam. Systems 18 no. 4, 889-935, (1998). 
[12] M. Jutila, On exponential sums involving the divisor function., J. Reine Angew. Math. 355, 173-190, (1985).

[13] M. Mirek and B. Trojan, Cotlar's ergodic theorem along the prime numbers, arXiv:1311.7572.

[14] P. Sarnak, Three lectures on the Mobius function randomness and dynamics, publications.ias.edu/sarnak/.

[15] E. M. Stein and N. J. Weiss, On the convergence of Poisson integrals, Trans. Amer. Math. Soc. 140 (1969), $35-54$.

[16] G. Tenenbaum, Introduction to analytic and probabilistic number theory. Translated from the second French edition (1995) by C. B. Thomas. Cambridge Studies in Advanced Mathematics, 46. Cambridge University Press, Cambridge, 1995. xvi+448 pp.

[17] M. Weber (2009) Dynamical Systems and Processes, European Mathematical Society Publishing House, IRMA Lectures in Mathematics and Theoretical Physics 14, xiii+759p.

[18] M. Wierdl, Pointwise ergodic theorem along the prime numbers, Israel J. Math. 64 no. 3, 315-336, (1989).

[19] J. R. Wilton, An approximate functional equation with applications to a problem of Diophantine approximation, J. für die reine und angewandte Math. 169, 219-237, (1933).

[20] A. Wintner, The theory of measure in arithmetical semi-groups, Wawerly Press, Baltimore, Md. (1944).

[21] A. Zygmund, (2002) Trigonometric series, Third Ed. Vol. 1\&2 combined, Cambridge Math. Library, Cambridge Univ. Press.

Ecole Centrale, Grande Voie des Vignes, 92290 Châtenay-Malabry, France

E-mail address: christophe.cuny@ecp.fr

IRMA, 10 rue du Général Zimmer, 67084 Strasbourg Cedex, France

E-mail address: michel.weber@math.unistra.fr 